\title{
Preexisting antibodies can protect against congenital cytomegalovirus infection in monkeys
}

Cody S. Nelson, ${ }^{1}$ Diana Vera Cruz, ${ }^{2}$ Dollnovan Tran, ${ }^{3}$ Kristy M. Bialas, ${ }^{1}$ Lisa Stamper, ${ }^{1}$ Huali Wu, ${ }^{4}$ Margaret Gilbert, ${ }^{3}$ Robert Blair, ${ }^{3}$ Xavier Alvarez, ${ }^{3}$ Hannah Itell, ${ }^{1}$ Meng Chen, ${ }^{1}$ Ashlesha Deshpande, ${ }^{5}$ Flavia Chiuppesi, ${ }^{6}$ Felix Wussow, ${ }^{6}$ Don J. Diamond, ${ }^{6}$ Nathan Vandergrift, ${ }^{1}$ Mark R. Walter, ${ }^{5}$ Peter A. Barry, ${ }^{7}$ Michael Cohen-Wolkowiez, ${ }^{4}$ Katia Koelle, ${ }^{2}$ Amitinder Kaur, ${ }^{3}$ and Sallie R. Permar ${ }^{1}$ Human Vaccine Institute, Duke University School of Medicine, and 'Department of Biology, Duke University, Durham, North Carolina, USA. ${ }^{3}$ Tulane National Primate Research Center, Tulane University, Covington, Louisiana, USA. ${ }^{4}$ Duke Clinical Research Unit, Duke University School of Medicine, Durham, North Carolina, USA. ${ }^{5}$ Department of Microbiology, University of Alabama, Birmingham, Alabama, USA. ${ }^{6}$ Department of Experimental Therapeutics, Beckman Research Institute of the City of Hope, Duarte, California, USA. ${ }^{\top}$ Center for Comparative Medicine, Department of Pathology and Laboratory Medicine, University of California, Davis, California, USA.

Human cytomegalovirus (HCMV) is the most common congenital infection and a known cause of microcephaly, sensorineural hearing loss, and cognitive impairment among newborns worldwide. Natural maternal HCMV immunity reduces the incidence of congenital infection, but does not prevent the disease altogether. We employed a nonhuman primate model of congenital CMV infection to investigate the ability of preexisting antibodies to protect against placental CMV transmission in the setting of primary maternal infection and subsequent viremia, which is required for placental virus exposure. Pregnant, $\mathrm{CD}^{+} \mathrm{T}$ cell-depleted, rhesus $\mathrm{CMV}$-seronegative (RhCMV-seronegative) rhesus monkeys were treated with either standardly produced hyperimmune globulin (HIC) from RhCMV-seropositive macaques or dose-optimized, potently RhCMV-neutralizing HIC prior to intravenous challenge with an RhCMV mixture. HIC passive infusion provided complete protection against fetal loss in both groups. The dose-optimized, RhCMV-neutralizing HIC additionally inhibited placental transmission of RhCMV and reduced viral replication and diversity. Our findings suggest that the presence of durable and potently neutralizing antibodies at the time of primary infection can prevent transmission of systemically replicating maternal RhCMV to the developing fetus, and therefore should be a primary target of vaccines to eliminate this neonatal infection.

Conflict of interest: S.R. Permar and A. Kaur provide consulting services to Pfizer Inc. for their preclinical human cytomegalovirus (HCMV) vaccine program and associated animal models.

Submitted: March 14, 2017

Accepted: May 23, 2017

Published: July 6, 2017

\section{Reference information:}

JCI Insight. 2017;2(13):e94002.

https://doi.org/10.1172/jci.

insight. 94002 .

\section{Introduction}

The current epidemic of Zika virus in the Americas has raised significant awareness of the societal and pediatric health impact of a congenitally transmitted neuropathogen. Yet nearly 1 million infants worldwide (1 out of every 150 live births) are born each year with congenital human cytomegalovirus (HCMV) infection $(1,2)$. Congenital HCMV, like congenital Zika virus, can cause microcephaly, hearing/vision loss, and abnormalities in nervous system development $(1,3)$. And though HCMV accounts for more congenital disease than all 29 newborn conditions currently screened for in the United States combined (4), public knowledge and effective interventions are severely lacking $(5,6)$. In recognition of this burden of disease, a congenital HCMV vaccine has remained a Tier 1 priority of the National Academy of Medicine for the past 17 years (7).

Drawing upon standard vaccine strategies, the HCMV field has previously attempted to induce potent antiviral immunity by various approaches including attenuation of live viruses (8-10), formulation of HCMV glycoprotein subunit vaccines (11-13), use of viral vectors for epitope expression (14-16), and delivery of single and bivalent DNA plasmid vaccines $(17,18)$. However, our understanding of anti-HCMV humoral immunity is complicated by the number of distinct glycoprotein complexes that contain neutralizing epitopes, most notably glycoprotein B (gB; fusion protein) and the pentameric complex gH/gL/UL128-131A (PC; necessary for entry into epithelial/endothelial cells) (19). While all vaccine platforms tested thus far ultimately failed 
to reach target endpoints in human clinical trials, the HCMV gB subunit vaccine demonstrated moderate ( $\sim 50 \%)$ efficacy at preventing primary HCMV infection, which is promising for future vaccine-development efforts $(12,13)$. Additional retrospective human studies have reported that neutralizing antibodies targeting HCMV surface glycoproteins are correlated with reduced incidence of congenital virus transmission after primary maternal HCMV infection (20-22). However, a recent clinical trial failed to demonstrate a significant reduction in rates of congenital HCMV infection following passive infusion of hyperimmune globulin (HIG) to pregnant women with primary HCMV infection. Results from these past studies emphasize possible challenges for the development of an efficacious antibody-based vaccine (23).

One major barrier the HCMV vaccine field has faced is the lack of a highly translatable animal model of congenital virus transmission. For the past 50 years, vaccine efficacy studies that evaluate protection against congenital HCMV transmission have been reliant upon either (a) small-animal models using species-specific viruses with limited HCMV sequence homology or (b) costly and arduous human clinical trials. We recently reported on the first nonhuman primate (NHP) model of congenital CMV transmission following primary infection of rhesus monkey dams with rhesus CMV (RhCMV) (24). There are numerous logistical advantages to an NHP model including enhanced immunologic tools (e.g., monoclonal antibodies) and extensive knowledge of immune cell subsets (e.g., cellular markers, B/T cell receptor repertoire databases). Furthermore, we hypothesize that this NHP model may more accurately reflect HCMV pathogenesis than small-animal models owing to increased similarity of host and virus. The rhesus monkey placenta is anatomically, physiologically, and immunologically quite similar to that in humans, which likely enhances the translational applications of the model (25). Additionally, RhCMV is genetically more homologous to HCMV than guinea pig or murine CMV $(24,26,27)$ and employs highly conserved immune-evasion pathways including viral IL-10 (28) and MHC downregulation (29).

Our previous study established that RhCMV could cross the placenta and cause congenital infection following intravenous (i.v.) inoculation of either immune-competent or $\mathrm{CD}^{+} \mathrm{T}$ cell-depleted seronegative dams during the second trimester of pregnancy. The i.v. route of RhCMV inoculation was selected for this experimental congenital infection model over mucosal routes of exposure, as it induced reproducible high levels of viremia and virus shedding in the urine and saliva of infected macaques, and hence best mirrored the prerequisite for systemic CMV replication prior to placental virus transmission in congenital CMV infection. In this study, we observed that $\mathrm{CD}^{+} \mathrm{T}$ cell-depleted dams frequently aborted their fetus following virus inoculation, exhibited higher plasma and amniotic fluid viral loads, and had delayed production of autologous neutralizing antibodies.

These results suggested that maternal humoral immunity may impact systemic and intrauterine RhCMV replication, thereby influencing the severity of congenital infection. As such, we sought to use this NHP model to investigate whether preexisting antibody alone, in the absence of virus-specific $\mathrm{T}$ cell responses, could protect against placental RhCMV transmission or reduce congenital RhCMV infection severity. The passive infusion of antibodies in this study was not intended to model a treatment strategy for the prevention of either maternal CMV acquisition or fetal virus transmission, but rather as a means to understand the protection conferred by preexisting maternal antibody. These insights will inform rational development of a vaccine to elicit the most critical immune responses required to eliminate congenital HCMV disease.

\section{Results}

$H I G$ production and study design. Two separate preparations of HIG were purified from plasma of RhCMVseropositive rhesus monkey donors. The first preparation (hereafter referred to as standard) was obtained from donors with high levels of total RhCMV-specific IgG, while the second preparation (hereafter referred to as high-potency) was purified from plasma of donors exhibiting robust epithelial cell-neutralizing IgG antibody titers $\left(\mathrm{ID}_{50}>1: 1,000\right)$. The high-potency preparation had approximately 4-fold more potent epithelial cell neutralization (standard $=22.6 \mu \mathrm{g} / \mathrm{ml}$ vs. high-potency $=5.9 \mu \mathrm{g} / \mathrm{ml}$ ) and slightly increased overall glycoprotein binding (RhCMV gB/RhCMV PC [RhgB/RhPC]: standard = 1.7/3.9 $\mu \mathrm{g} /$ $\mathrm{ml}$ vs. high-potency $=1.0 / 3.1 \mu \mathrm{g} / \mathrm{ml}$ ) versus the standard preparation. All RhCMV-seronegative dams in our current and previous study were infused with a $\mathrm{CD}^{+} \mathrm{T}$ cell-depleting antibody at week 7 of gestation, as previously described (24). T cell phenotyping (Supplemental Figure 1 and Supplemental Table 1; supplemental material available online with this article; https://doi.org/10.1172/jci.insight.94002DS1) revealed a decline in $\mathrm{CD}^{+} \mathrm{T}$ cells with changes in the $\mathrm{CD}^{+} \mathrm{T}$ cell population in response to primary RhCMV infection (Supplemental Figure 2). The dams were subsequently divided into 3 groups: control, 


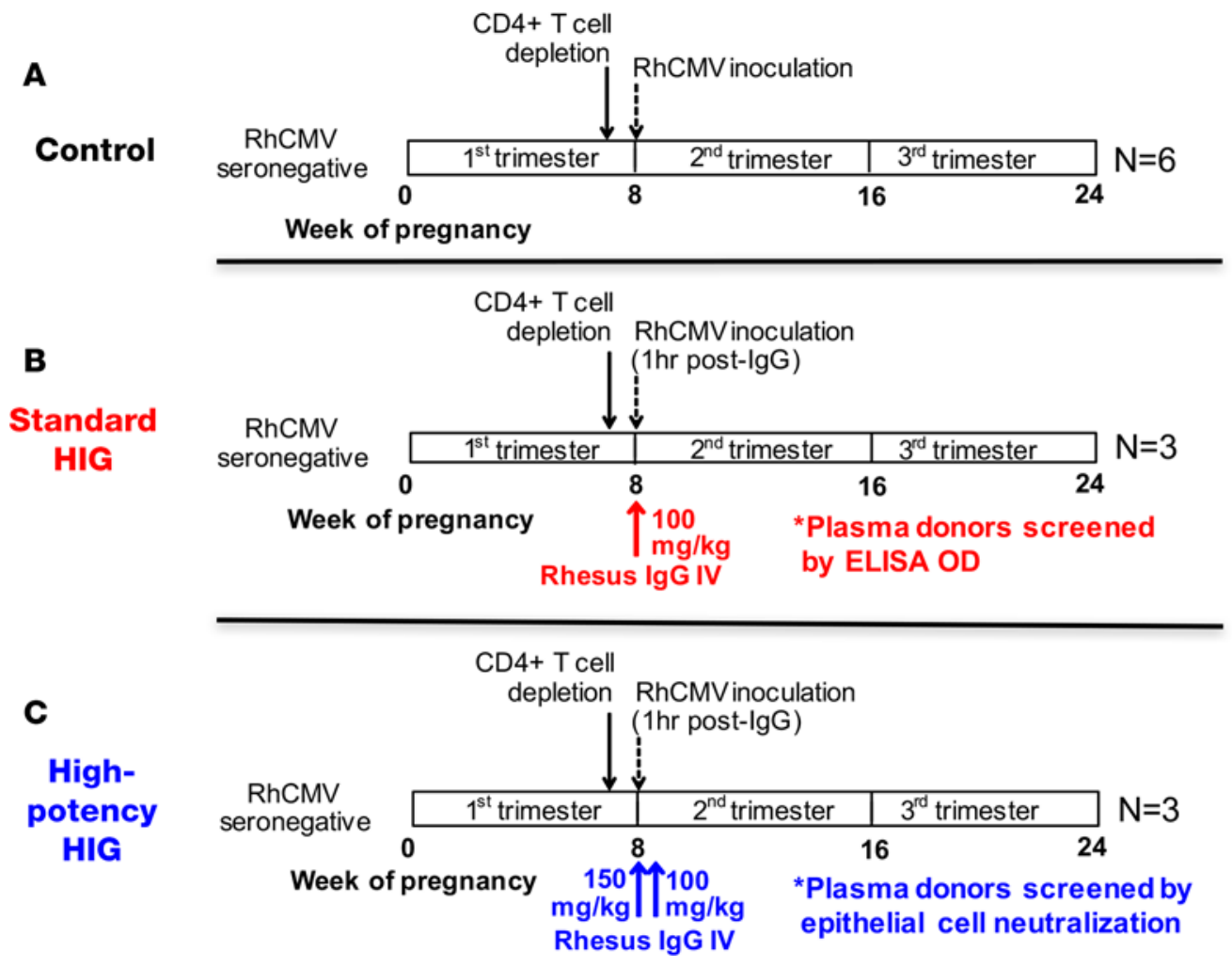

Figure 1. HIG preinfusion/RhCMV placental transmission study experimental design. (A) Six rhesus CMV-seronegative (RhCMV-seronegative) rhesus monkey dams were CD4+ $T$ cell depleted at week 7 of gestation and then inoculated 1 week later with a mixture of RhCMV stocks (180.92, UCD52, and UCD59). (B) Three seronegative, $\mathrm{CD} 4^{+} \mathrm{T}$ cell-depleted dams received a single dose $(100 \mathrm{mg} / \mathrm{kg})$ of a standard hyperimmune globulin (HIC) preparation 1 hour prior to RhCMV infection. (C) Three seronegative, $\mathrm{CD}^{+} \mathrm{T}$ cell-depleted dams received a dose-optimized HIG regimen (150 mg/kg prior to RhCMV infection $+100 \mathrm{mg} / \mathrm{kg}$ 3 days following infection) of a high-neutralizing-potency HIG preparation isolated from seropositive donors screened by epithelial cell neutralization.

standard, and high-potency. Each animal, including those in the control group $(n=6$, including 4 historical controls; see ref. 24) were i.v. inoculated with a mixture of fibroblast-tropic 180.92 (30) and epithelial cell-tropic UCD52/UCD59 (31) RhCMV viruses at week 8 of gestation (Figure 1A). The standard group $(n=3)$ was administered a single dose $(100 \mathrm{mg} / \mathrm{kg}$, which mimics a previously published human trial protocol; see ref. 23) of the standard HIG preparation 1 hour prior to inoculation with the RhCMV variants (Figure 1B), while the high-potency group $(n=3)$ was given a dose-optimized regimen of the highpotency HIG preparation (Supplemental Figure 3$) 1$ hour prior to $(150 \mathrm{mg} / \mathrm{kg}$ ) and 3 days following (100 $\mathrm{mg} / \mathrm{kg}$ ) RhCMV inoculation (Figure 1C and Supplemental Figure 4).

Glycoprotein targets of RhCMV-neutralizing antibodies. To determine the RhCMV antibody specificity of the administered HIG, both standard (Figure 2A) and high-potency HIG (Figure 2B) were depleted for antibodies specific for RhgB, RhPC, as well as RhgB/RhPC combined. Sufficient and selective depletion of glycoproteinspecific antibodies was confirmed by ELISA against the depleted epitope. We confirmed a greater than $75 \%$ decrease in $\mathrm{EC}_{50}$ magnitude against depleted epitope and less than $20 \%$ change in $\mathrm{EC}_{50}$ against nondepleted epitope (Table 1). For the standard HIG preparation, adsorption with RhgB resulted in a 2.2-fold reduction in the neutralization potency of plasma (mock-depleted $\mathrm{IC}_{50}=32.3 \mathrm{vs}$. RhgB-depleted $\mathrm{IC}_{50}=71.8$ ), whereas adsorption with $\mathrm{RhPC}$ resulted in a slightly higher 2.9-fold reduction in neutralization potency (RhPC-depleted $\left.\mathrm{IC}_{50}=94.6\right)$ (Figure $2 \mathrm{~A}$ and Table 1). This same trend was observed for antigen depletion of the high-potency HIG, with a 2.9-fold reduction following RhgB depletion and a 3.3-fold reduction following RhPC depletion (Figure 2B and Table 1). Additionally, depletion of antibodies specific for both antigens reduced the neutralization potency of sera beyond that of depletion with either RhgB or RhPC alone (Figure 2, A and B, and Table 1). Altogether, these findings suggest that antibodies targeting both RhgB and RhPC contribute to overall RhCMV neutralization activity in roughly equivalent proportions. Furthermore, both RhgB- and RhPC-specific antibodies were found to contribute to the RhCMV-neutralization titer in individual RhCMV-seropositive rhesus monkeys (Supplemental Figure 5). 
A

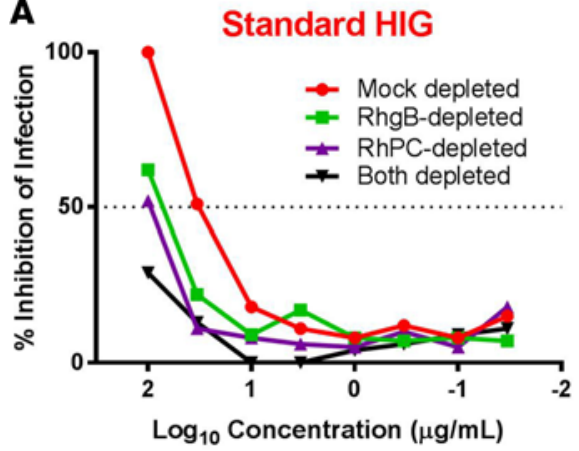

B

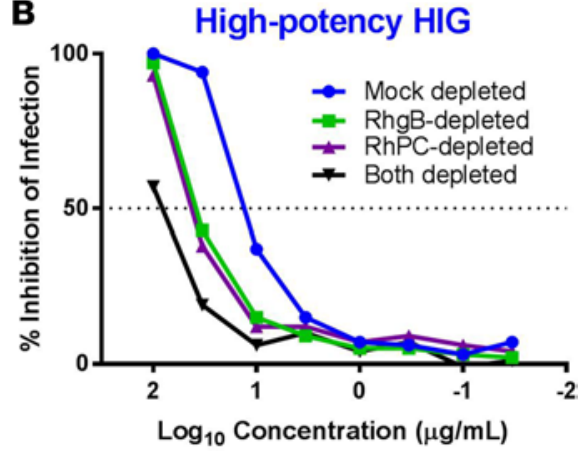

Figure 2. Both RhCMV gB- and PC-specific antibodies contribute to neutralization activity of purified HIC preparations. Neutralization curves for standard hyperimmune globulin (HIG) (A) and high-potency HIG (B) compare the relative neutralization potency of mock-depleted, rhesus CMV (RhCMV) glycoprotein B-depleted (RhgB-depleted), RhCMV pentameric complex-depleted (RhPC-depleted), and both RhgB/RhPC-depleted antibody preparations. Each curve represents the mean value of 2 experimental replicates.

Antibody kinetics following HIG infusion. Total RhCMV-binding IgM and IgG titers (Figure 3, A and B), RhgB- and RhPC-specific IgG titers (Figure 3, C and D), and fibroblast/epithelial cell neutralizing antibody titers (Figure 3, E and F), were measured following HIG infusion. Based on the known kinetics of antibody responses to primary viral infections, we attributed early detection of RhCMV-specific activity (within the first 10 days) to reflect passively infused antibodies in the HIG preparations. Peak RhCMVbinding IgG as well as RhgB- and RhPCspecific antibody titers in the high-potency HIG group surpassed those of the standard HIG group (Figure 3, B-D), while peak neutralization titers were similar between the 2 HIG groups (Figure 3, E and F). However,

levels of IgG binding and antibody neutralization were sustained near peak levels for 10 days in the doseoptimized, high-potency HIG group compared with only 3 days in the standard HIG group (Figure 3, B-E). It is noteworthy that average binding-IgG and epithelial cell-neutralization titers in the high-potency HIG group surpassed those of chronically infected rhesus monkeys (Figure 3, B and E, and Supplemental Figure 5) (24). The standard HIG group had the most robust natural host immune response, with a sustained elevation of RhCMV-specific IgM (Figure 3A), as well as an exponential rise in binding-IgG (Figure 3B) and neutralization titers (Figure 3, D and E) that outpaced each of the other groups.

RhCMV viral load, placental transmission, and shedding. Following RhCMV inoculation, both control and standard HIG group animals showed a rapid onset of viremia that peaked at 2 weeks after infection (Figure $4 \mathrm{~A})$. In contrast, peak viremia in the high-potency HIG group was delayed until 4 weeks after infection (Figure 4A), with a median value nearly 2 logs lower than the control group (Figure 4E; $P=0.047$, corrected Wilcoxon exact test). RhCMV DNA copy number in amniotic fluid was used as a marker for placental virus transmission because it is the gold-standard clinical test for congenital HCMV infection (32). All 6 control animals had detectable RhCMV DNA in amniotic fluid between 1 and 3 weeks following infection (Figure 4B), indicating 100\% placental transmission. Additionally, 2 of 3 animals in the standard HIG group had detectable congenital RhCMV infection (67\% transmission) (Figure 4B). However, none of the 3 animals in the high-potency HIG group had detectable RhCMV DNA in the amniotic fluid, suggesting complete inhibition of congenital RhCMV transmission (Figure 4B), and indicating a potential dose effect of HIG-mediated protection. It is possible that systemic viral load influenced placental transmission, as animals that transmitted the virus had the highest peak plasma viral loads (Figure 4E) and peak plasma viral load correlated with initial amniotic fluid viral load (Figure 4F; $r=0.812, P=0.001$, Spearman correlation). Finally, while there was no difference in the peak magnitude of viral shedding in maternal urine

Table 1. Antigen-binding and neutralization activity of RhgB- and RhPC-depleted plasma

\begin{tabular}{|c|c|c|c|c|c|c|}
\hline & \multicolumn{3}{|c|}{ Standard HIG } & \multicolumn{3}{|c|}{ High-potency HIG } \\
\hline & $\begin{array}{l}\text { RhgB EC }_{50} \\
(\mu \mathrm{g} / \mathrm{ml})^{A}\end{array}$ & $\begin{array}{l}\mathrm{RhPC}_{\mathrm{EC}} \\
(\mu \mathrm{g} / \mathrm{ml})^{A}\end{array}$ & $\begin{array}{c}\mathrm{IC}_{50} \\
(\mu \mathrm{g} / \mathrm{ml})\end{array}$ & $\begin{array}{l}\text { RhgB EC }_{50} \\
(\mu \mathrm{g} / \mathrm{ml})^{A}\end{array}$ & $\begin{array}{c}\mathrm{RhPC} \mathrm{CC}_{50} \\
(\mu \mathrm{g} / \mathrm{ml})^{A}\end{array}$ & $\begin{array}{c}\mathrm{IC}_{50} \\
(\mu \mathrm{g} / \mathrm{ml})\end{array}$ \\
\hline Undepleted & 1.7 & 3.9 & 22.6 & 1.0 & 3.1 & 5.9 \\
\hline Mock-depleted & 5.9 & 15.7 & 32.3 & 3.1 & 9.0 & 13.1 \\
\hline RhgB-depleted & $>33.3^{B}$ & 17.7 & 71.8 & 10.9 & 7.1 & 38.3 \\
\hline RhPC-depleted & 5.26 & $>33.3^{B}$ & 94.6 & 3.8 & $>33.3^{B}$ & 42.6 \\
\hline Both depleted & $>33.3^{B}$ & $>33.3^{B}$ & $>100^{c}$ & 15.3 & $>33.3^{B}$ & 82.5 \\
\hline
\end{tabular}

${ }^{A} \mathrm{EC}_{50}$ values measured in $\mu \mathrm{g} / \mathrm{ml}$ of HIG equivalent. ${ }^{B}$ Starting ELISA concentration of $33.3 \mu \mathrm{g} / \mathrm{ml}$. ${ }^{\mathrm{C} S t a r t i n g}$ neutralization concentration of $100 \mu \mathrm{g} / \mathrm{ml}$. HIG, hyperimmune globulin; RhgB, rhesus CMV glycoprotein B; RhPC, rhesus CMV pentameric complex. 

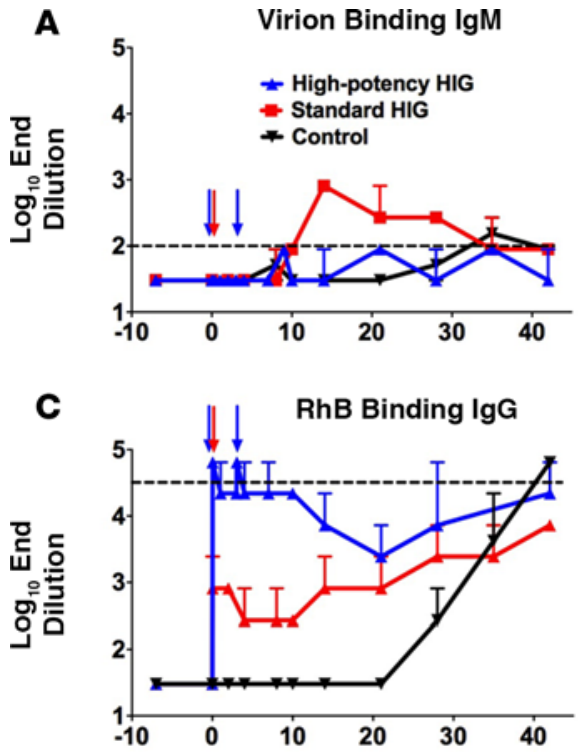

E

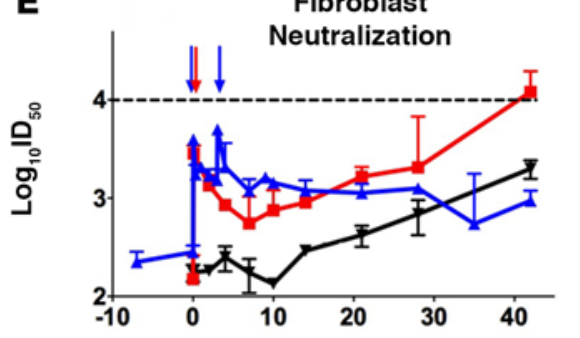

B

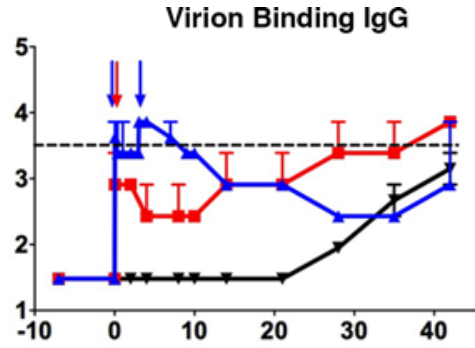

D

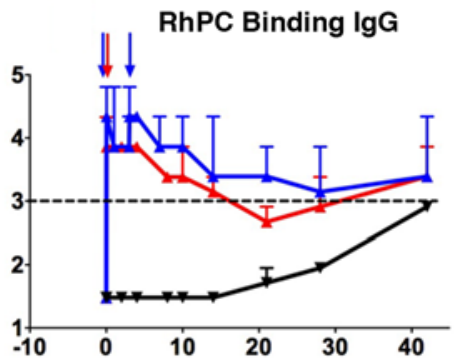

$\mathbf{F}$

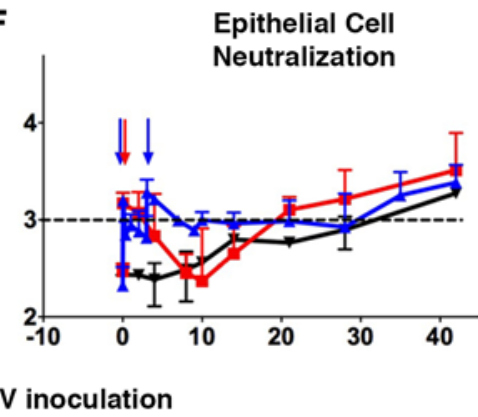

Figure 3. Plasma RhCMV IgM/IgG binding and neutralization following HIC infusion. Median whole UCD52 rhesus CMV (RhCMV) virion IgM

(A) and IgG (B) binding titers are shown for 2 control animals in black (excluding 4 historical controls because of sample limitation), the 3 animals infused with a single dose of standard hyperimmune globulin (HIG) in red, and the 3 animals administered a dose-optimized regimen of high-potency HIC in blue. RhCMV glycoprotein $\mathrm{B}$ (RhgB) (C) and RhCMV pentameric complex (RhPC) (D) binding IgG titers demonstrate higher glycoprotein binding in the high-potency HIGinfused group. Furthermore, median neutralization titers $\left(I_{50}\right)$ were measured on fibroblasts (180.92 virus) (E) and epithelial cells (UCD52 virus) (F). The time points of HIG infusion are denoted by colored arrows. Two experimental replicates were averaged to obtain each value, and then the median and numerical range was plotted for animals within the same treatment group. Horizontal dotted lines indicate average antibody-binding and neutralization titers for chronically RhCMV-infected rhesus monkeys.

\section{Days after RhCMV inoculation}

(Figure 4C) or saliva (Figure 4D) between treatment groups, there was delayed onset of shedding in both urine (Figure 4G) and saliva (Figure $4 \mathrm{H}$ ) following high-potency HIG infusion (both $P=0.047$, corrected Wilcoxon exact test). Altogether, these findings suggest that the presence of neutralizing antibodies can reduce systemic replication and block dissemination of the virus to other anatomic compartments.

Short NGS amplicon population profiling (SNAPP) of plasma virus. Using a validated (Supplemental Figure 6) next-generation sequencing (NGS) technique, short 400-bp regions within the RhCMV gB (Figure 5) and gL (Supplemental Figure 7) genetic loci were amplified and sequenced at great read depth. We found that, as previously reported (24), the viral populations in plasma were composed primarily of strain UCD52 at each sampled time point and in all treatment groups. The reason for the consistent predominance of UCD52 over other viral strains 180.92/UCD59 is unclear, though may indicate enhanced in vivo fitness of this isolate. Additionally, viral diversity was measured by calculating mean nucleotide diversity $(\pi)$ across each sample's haplotype sequences (Supplemental Table 2). Overall, the diversity of the dominant plasma UCD52 viral subpopulation was restricted in the high-potency HIG-treated animals compared with the control group at both the gB (Figure 5C) and gL (Supplemental Figure 7) loci (both $P=0.036$, Wilcoxon exact test). Nucleotide diversity was not significantly different between the control and standard HIG groups.

Fetal outcome and RhCMV congenital infection. While 5 of 6 control dams aborted their fetus at 3 weeks after RhCMV infection, administration of either HIG regimen was sufficient to protect fetuses from spontaneous abortion ( $P=0.015$, exact log-rank test based on Heinze macro; Figure 6A) (33). Furthermore, we observed a reduced rate of congenital RhCMV transmission among HIG-treated animals (2 of 6) compared with control animals (6 of 6$)(P=0.049$, exact log-rank test based on Heinze macro; Figure 6B) (33). Intriguingly, in the standard HIG group only 1 of 3 fetuses remained uninfected, whereas all 3 fetuses in the high-potency HIG group were protected from infection (Figure 6C), suggesting that preexisting, potently CMV-neutralizing HIG can reduce the incidence of congenital infection. Of note, fetal growth curves (Supplemental Figure 8) and systemic cytokine profiles (Supplemental Figure 9) were similar between treatment 

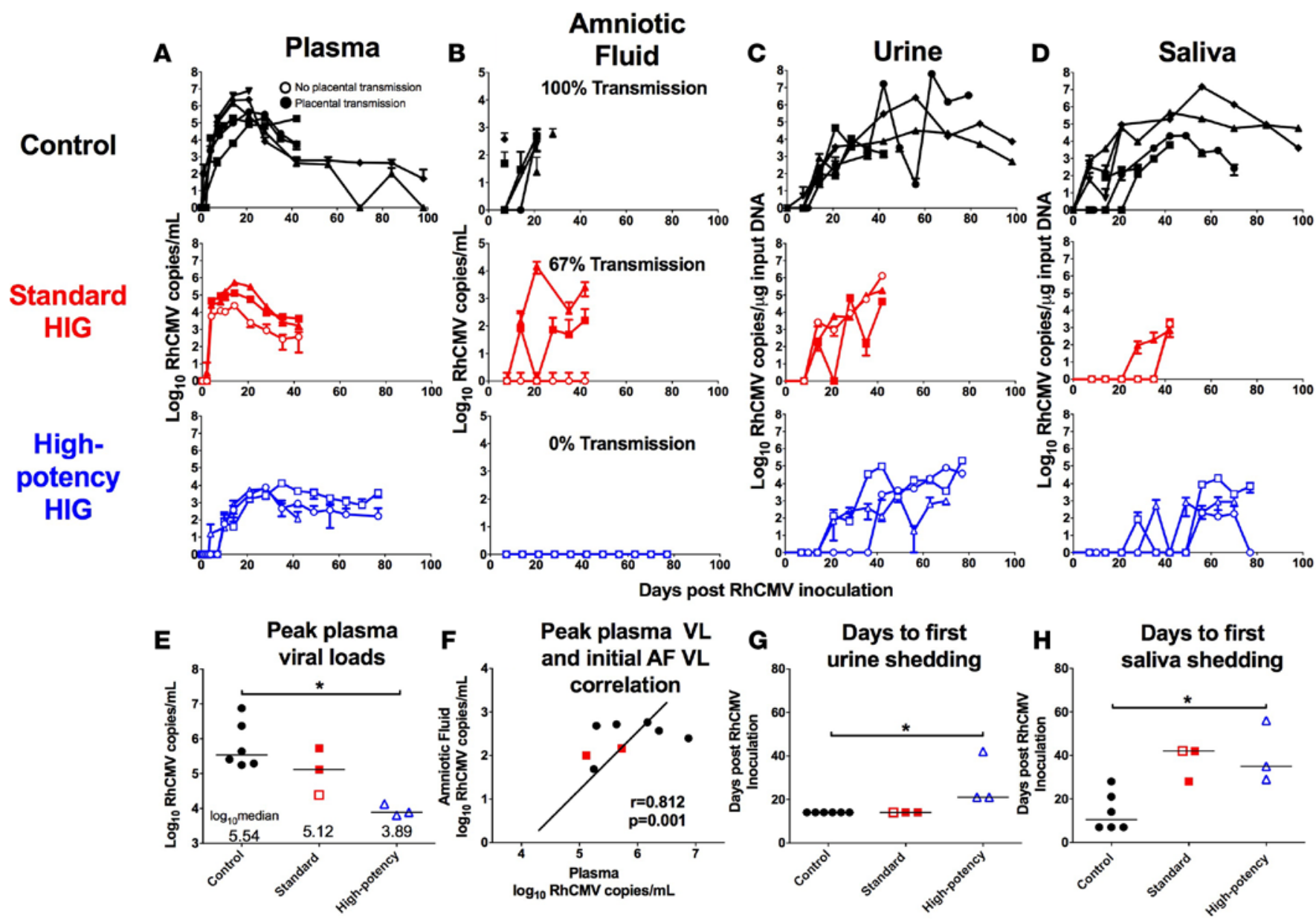

Figure 4. Infusion with high-potency HIG prior to RhCMV infection reduces plasma viral load, prevents placental virus transmission, and delays viral shedding. Rhesus CMV (RhCMV) copy number determined by real-time PCR is shown for control animals ( $n=6$; black), the standard hyperimmune globulin (HIG) group ( $n=3$; red), and the high-potency HIG group ( $n=3$; blue) in plasma (A), amniotic fluid (B), urine (C), and saliva (D). Data shown as the mean value of 6 or more individual replicates, with error bars indicating SD. Filled symbols represent animals with placental transmission, and nonfilled symbols represent those without transmission. (E) Compared with the control group, median peak plasma viral load (VL) is reduced by nearly 2 logs in the high-potency HIG group ( $P=0.047$, corrected Wilcoxon exact test). (F) There is a correlation between peak maternal plasma VL and initial amniotic fluid (AF) VL ( $r=0.812, P=0.002$, nonparametric Spearman correlation). Additionally, in comparison with the control group, the average number of days to first urine shedding $(\mathbf{C})$ and first saliva shedding $(\mathbf{H})$ is increased in the high-potency HIG group (both $P=0.047$, corrected Wilcoxon exact test). Horizontal bars indicate median values for each group. ${ }^{*} P<0.05$, corrected Wilcoxon exact test.

groups, indicating no obvious off-target effects of the infused HIG. Reassuringly, all dams with detectable RhCMV DNA in amniotic fluid also had detectable virus in placenta, amniotic membrane, and umbilical cord (Figure 6C). However, there was significant heterogeneity in viral burden and distribution among fetal tissue types; some fetuses had high copy number virus in nearly every tissue tested (174-97, IM67), while others only had virus detectable in the cochlea (GI73, HD79) (Figure 6C). This heterogeneity is consistent with previous reports for HCMV suggesting that the virus can replicate in the amniotic membrane without observable fetal infection (34). It is unclear from the data whether cochlear infection might have occurred via direct inoculation of the ear canal or if fetal immune control could have led to isolated virus detectable in the cochlea. However, the persistence of virus in the cochlea further validates this model as consistent with congenital HCMV infection in human fetuses. Congenital infection was additionally confirmed in placentas by histology and immunohistochemical staining for the RhCMV IE-1 protein (Figure 6D and Supplemental Table 3). Of note, the placental tissue PCR and histology/IHC data do not agree completely (Supplemental Table 3), suggesting that PCR may be a more sensitive measure after resolution of the acute infection.

Placental transcriptome. Congenital RhCMV infection appears to modify the placental transcriptome more radically than HIG infusion or fetal abortion, as more than 300 genes were differentially expressed between RhCMV-transmitting and nontransmitting dams, but only 78 when comparing HIG infusion/fetal outcome 


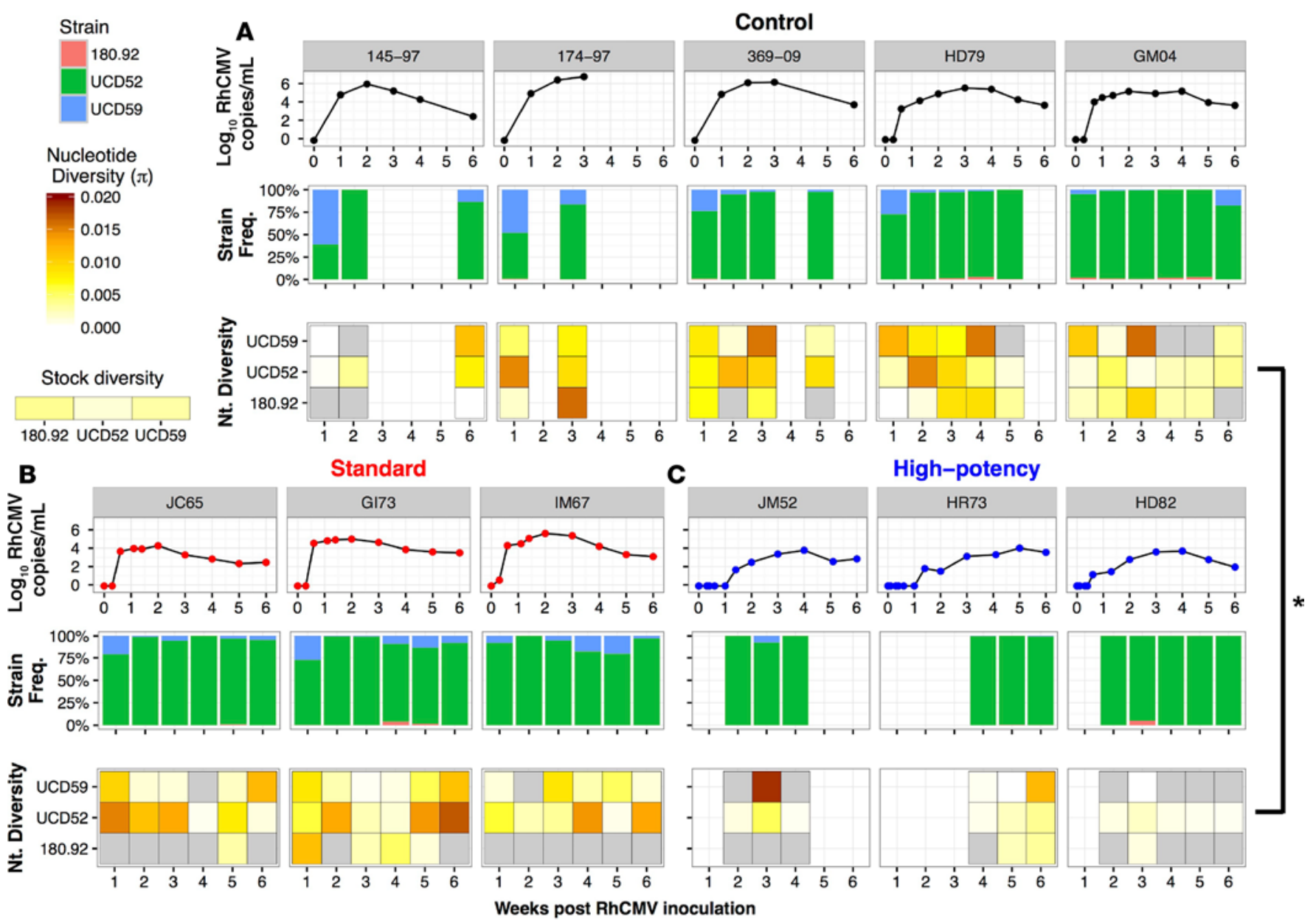

Figure 5. Plasma RhCMV gB sequence diversity is decreased following high-potency HIG infusion. SNAPP deep sequencing analysis at the rhesus CMV (RhCMV) glycoprotein B (gB) locus for maternal plasma virus population is shown for (A) 5 control dams (145-97, 174-97, 369-09, GM04, and HD79), (B) 3 standard hyperimmune globulin-infused (HIG-infused) dams (GI73, IM67, and JC65), and (C) 3 high-potency HIG-infused dams (HD82, HR73, and JM52). The top panel for each animal indicates the plasma viral load. The middle panel indicates the percentage of sequence reads corresponding to each of the 3 inoculated RhCMV strains in plasma at weeks 1-6 after infection (180.92 in pink, UCD52 in green, and UCD59 in blue), which was similar between the treatment groups. The bottom panel for each animal is a heatmap depiction of viral nucleotide diversity $(\pi)$. Scale ranges from white $(\pi=0)$ to dark red $(\pi=0.02)$, with the diversity of each viral stock displayed for reference. Gray coloring indicates that no sequence reads were detected at that time point for a given viral variant. Blank areas represent sample nonavailability (control group) or limited plasma viral load ( $<100$ copies $/ \mathrm{ml})$. There is a significant reduction in diversity at the $\mathrm{gB}$ locus for the dominant strain in plasma (UCD52) following high-potency HIG infusion ( ${ }^{*} P=0.036$, Mann-Whitney $U$ test). Each data point represents the mean value of 2 or more experimental replicates. Historical control animal 274-98 (24) was omitted from this analysis because it was only inoculated with a single strain (180.92).

(Figure 7A and Supplemental Figure 10). This finding suggests that HIG predominantly mediated protection through its impact on maternal systemic virus replication rather than through modification of the placental transcriptome. A large proportion of these differentially expressed genes were immune related. Additional cellular functions that appeared altered by RhCMV infection included metabolism, oxidoreductase activity, and cellular growth/proliferation (Supplemental Table 4). Gene interaction analysis of differentially expressed genes between RhCMV-transmitting and nontransmitting dams (Figure 7B) revealed that the placental transcriptome was heavily biased towards upregulation of genes in transmission. Furthermore, certain gene nodes had numerous connections to other differentially regulated genes (e.g., VCAM1, EGFR, GZMB, LYN, FYN, and GAPDH), suggesting that these keystone genes are central to the cellular pathways induced by RhCMV infection. Focusing on a subset of genes involved in innate versus adaptive immunity (Figure 7C), we note that there is a potential bias for activation of innate immune pathways by placental RhCMV infection. Finally, cell-type enrichment analysis based upon previously described genes involved in cellular activation/processes (35) demonstrated no preference at the transcriptome level for myeloid versus lymphoid lineages (Figure 7D). However, NK cell-specific genes had the most robust differential expression in RhCMV infection (Figure 7D), 

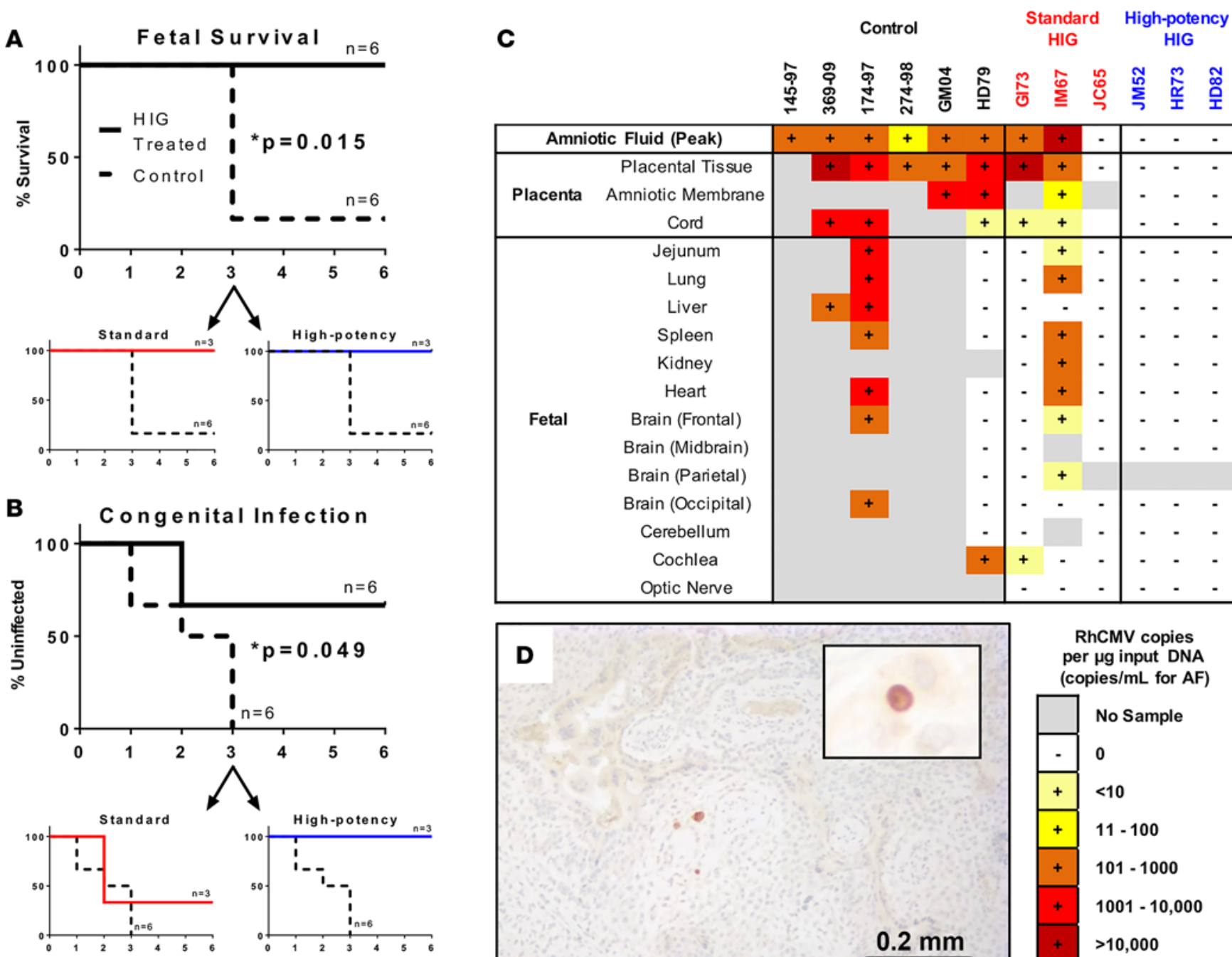

Weeks post RhCMV inoculation

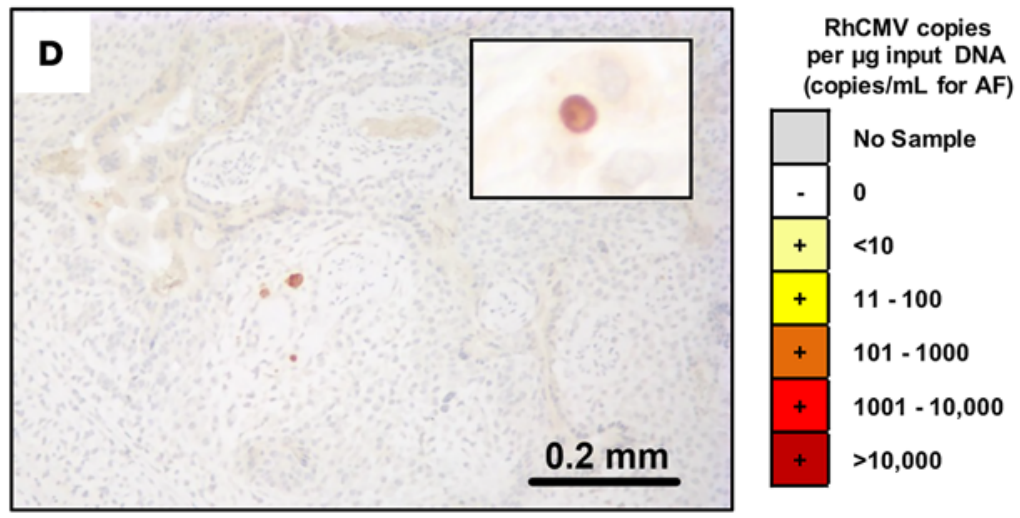

Figure 6. Maternal HIG preinfusion significantly reduces incidence and severity of rhesus CMV (RhCMV) congenital infection. (A) Fetal survival was significantly increased following hyperimmune globulin (HIG) infusion ( $P=0.015$, exact log-rank test based on Heinze macro), and both standard HIG and high-potency HIG infusion were sufficient to prevent fetal loss. (B) Rate of congenital infection was significantly reduced following HIG preinfusion ( $P=0.049$, exact log-rank test based on Heinze macro), though 2 of 3 animals in the standard HIG group had congenitally infected infants compared with 0 of 3 in the high-potency HIG group. (C) Heatmap separated by treatment group demonstrates heterogeneity in the viral burden of amniotic fluid (AF), placenta, amniotic membrane, cord, and fetal tissues. Data shown as the mean value of 6 or more individual replicates. Samples were considered positive if viral DNA was detected in 2 or more individual replicates. (D) Placental infection was detected by immunohistochemistry for RhCMV IE-1 protein, with rare cells in the trophoblastic shell of IM67 (standard HIG) exhibiting intranuclear staining. Inset: higher magnification of cell exhibiting intranuclear staining.

and we identified 21 unique genes known to be associated with NK cell function and/or chemotaxis (Figure 7E and Supplemental Table 5) including killer immunoglobulin-like receptors (KIR2DL4) and killer lectin-like receptors (KLRD1, KLRC1, KLRC3, and KLRB1). Furthermore, nonclassical MHC molecules HLA-E and HLA-G (rhesus analog inferred by IPA database) were upregulated.

\section{Discussion}

The role of preexisting antibodies in prevention of congenital HCMV infection remains controversial within the HCMV vaccine field, yet our findings indicate that a dose-optimized preinfusion with potently neutralizing HIG can block placental RhCMV transmission in a rhesus monkey model of congenital CMV. Our study illustrates that factors such as maternal antibody titer and neutralization potency at the time of peak CMV viremia are important considerations for antibody-mediated protection against congenital CMV infection. In this study, given the experimental setup, we have not definitively proven that 
A

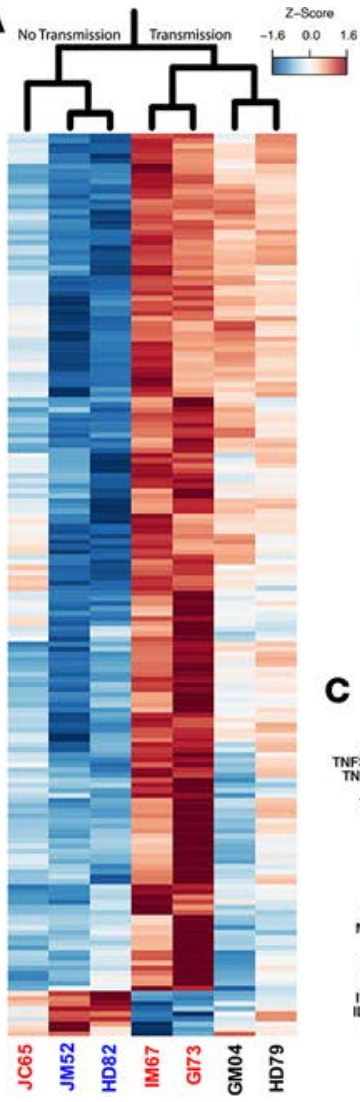

B
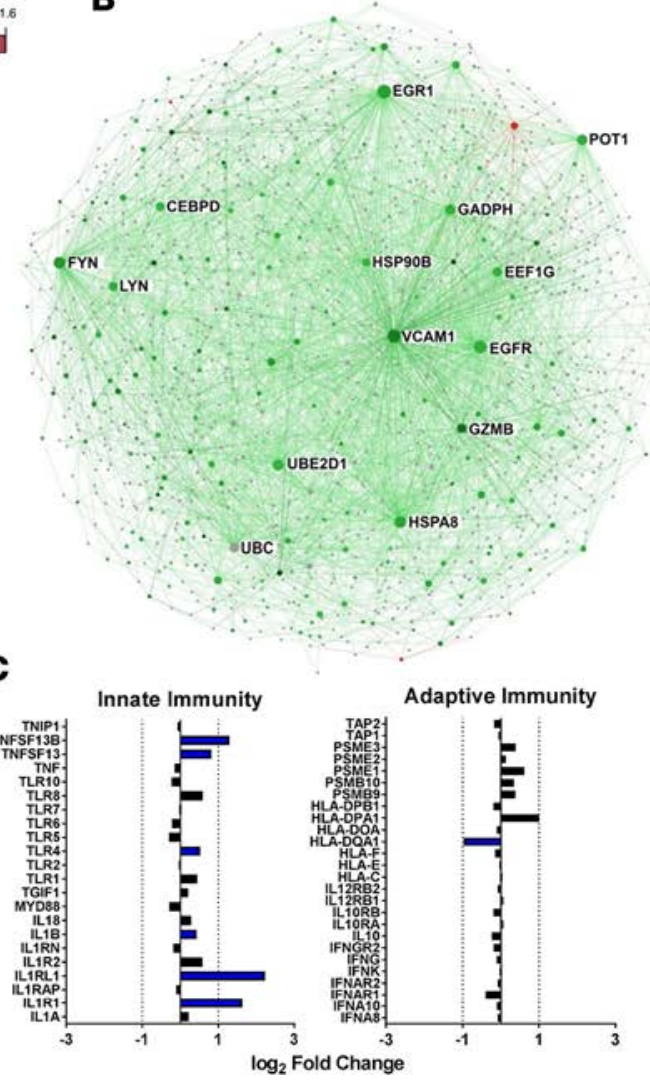

D
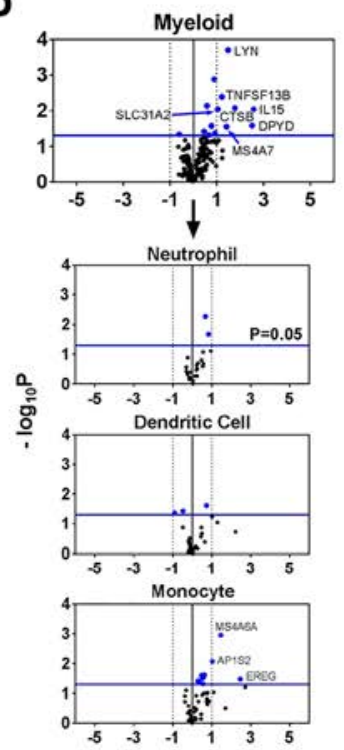

$\log _{2}$ Fold Change

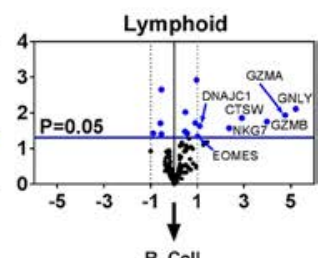

$\mathbf{E}$
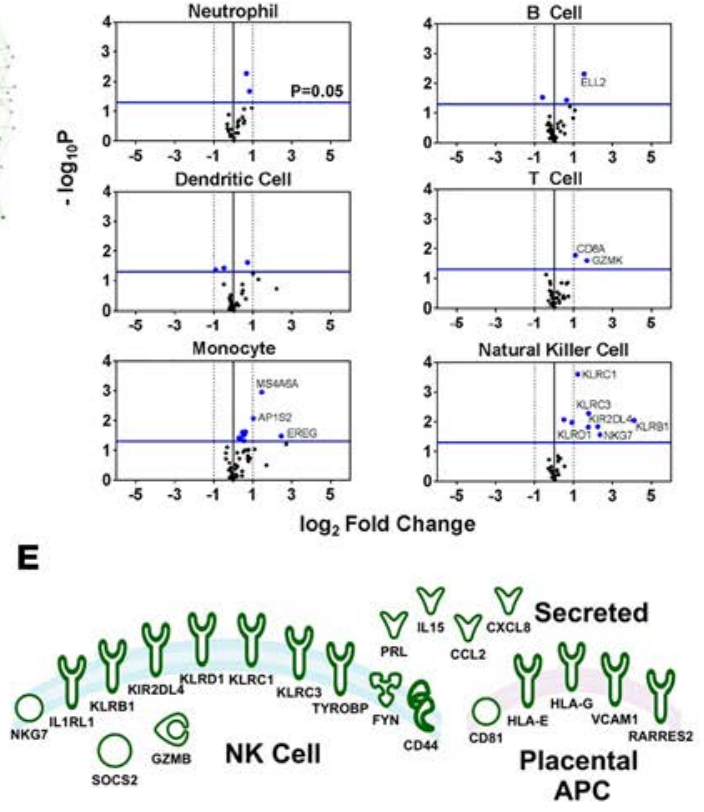

Figure 7. Placental transcriptome shifts following RhCMV congenital infection. (A) Heatmap depicting normalized fold change of top differentially expressed genes $(P<0.01$, fold change $>2.0)$ in monkey dam placenta with and without rhesus CMV (RhCMV) congenital infection (red $=$ relative upregulation in RhCMV infection, blue = relative downregulation). (B) Interaction network for all differentially regulated genes $(P<0.05$, fold change $>$ 2.0) suggests that genes upregulated in congenital infection (green) greatly outnumber those upregulated in no infection (red). Node size reflects the number of gene interactions (InnateDB database), while color intensity indicates degree of fold change. (C) Analysis of innate versus adaptive immune genes suggests some preference for upregulation of innate immunity following infection, with blue denoting a statistically significant change $(P<$ 0.05). (D) Cell-type enrichment analysis suggests no preference for myeloid versus lymphoid lineages, but that genes specific for monocytes and NK cells are upregulated following congenital infection. Statistically significant genes $(P<0.05)$ shown in blue, and significant genes with a fold change greater than 2.0 are labeled. (E) Diagram depicting placental genes related to NK cell movement and/or function (defined by IPA and KEGG databases) that are differentially upregulated $(P<0.05$, fold change $>2)$ in placental RhCMV infection. APC, antigen-presenting cell. Data represent values obtained from a single transcriptome microarray experiment.

it is neutralization titer that is protective against congenital RhCMV transmission. Other possibilities include the total preexisting binding-antibody level, non-neutralizing antibody functions such as antibody-dependent cellular cytotoxicity (ADCC) activity, a reduction in maternal viral load, or reduction in the number of infected monocytes that might traffic to the placenta. Nevertheless, these data substantiate observational human cohort studies suggesting that maternal HCMV-specific antibodies decrease risk of placental virus transmission (20-22, 36). Furthermore, these data affirm findings from the guinea pig model of congenital infection that demonstrate that preexisting antibodies (from either glycoprotein immunization or passive HIG/monoclonal antibody infusion) can reduce the incidence and/or severity of congenital guinea pig CMV infection (37-40).

The therapeutic efficacy of HIG in preventing placental transmission in mothers with primary HCMV infection is a topic of ongoing investigation. Several nonrandomized small-scale clinical trials $(41,42)$ and case-controlled studies $(43,44)$ concluded that postinfection HIG infusion significantly reduced rates of placental HCMV transmission, reduced placental pathology, and improved infant outcomes. Animal studies in the guinea pig model of congenital infection further corroborated these findings $(39,45)$. However, a large-scale, randomized, placebo-controlled trial did not find significant reduction in rates of congenital infection in mothers with primary HCMV infection that were subsequently treated with HIG, and revealed no difference in virus-specific antibody titers, effector $\mathrm{T}$ cell count, or the level of plasma viremia 
between the HIG-treated and placebo groups (23). Several confounding factors may explain the lack of efficacy of HIG treatment in this large-scale clinical trial. First, it was unknown in this study whether congenital transmission had already occurred prior to initiating therapeutic HIG treatment. Second, there is no concrete evidence for the therapeutic efficacy of the clinically utilized product (Cytotect), and therefore it is unknown whether HIG infusion achieved a titer that was both sustained and/or sufficient for effective anti-HCMV immunity.

This study was not intended to model a therapeutic treatment regimen but rather to directly address the clinical question, can preexisting antibodies alone prevent congenital CMV infection? The advantages of our NHP model are that the timing of infection is predefined and serum antibody titer can be titrated. By utilizing passive infusion experiments with different dosing regimens and potency of RhCMV-specific antibodies prior to RhCMV inoculation, we were able to model the requirements for preexisting antibodies to confer protection against congenital RhCMV infection. Moreover, conducting the experiments in a $\mathrm{CD}^{+} \mathrm{T}$ cell-depletion model also allowed us to address the protective role of antibodies to the exclusion of RhCMV-specific cellular immune responses. Our results suggest that a single $100 \mathrm{mg} / \mathrm{kg}$ dose of standard neutralizing potency HIG (donor plasma screened by elevated ELISA OD, as is done for the clinical product Cytotect; see ref. 46) delivered immediately prior to RhCMV infection can reduce the severity of congenital infection ( 0 of 3 monkeys aborted) but is insufficient to prevent congenital CMV transmission ( 2 of 3 monkeys transmitted).

To our knowledge, the epitope specificity of RhCMV-neutralizing antibodies has not been previously reported. It has been well established for HCMV that, while gB is an important target of neutralizing antibodies, the majority of neutralization activity in seropositve individuals is directed against the $\mathrm{gH} / \mathrm{gL} /$ UL128-131A pentameric complex $(47,48)$. Furthermore, the pentameric complex has also been shown to be a target of neutralizing antibodies for guinea pig CMV (49). In this study, we determined that antibodies targeting both RhgB and RhPC contribute to the total neutralization activity of RhCMV-seropositive monkey sera in approximately equivalent proportions (Figure 2). It is certainly reassuring for the translational applicability of this animal model system that RhPC-directed antibodies are neutralizing; however, it is unclear why the relative proportions of PC-directed neutralization activity would differ between HCMV ( 85\%) (47) and RhCMV ( $50 \%)$. We hypothesize that this could explain why the magnitude of RhCMV-neutralizing antibody titers are roughly equivalent in magnitude when measured in both fibroblasts and epithelial cells (Figure 3), whereas HCMV-neutralizing antibody titers are nearly an order of magnitude lower in fibroblasts than epithelial cells (50). Subsequent studies are needed to improve our understanding of this potential difference in viral biology and/or antiviral humoral immune factors between RhCMV and HCMV.

It has been hypothesized that passively infused antibodies can reduce systemic maternal viral load and thereby reduce the likelihood of placental transmission $(20,41)$, though this correlation has not been observed in some human primary infection cohorts (51). In this study, dams with reduced peak plasma viral load in the presence of antibodies did not transmit the infection to their fetus in utero (Figure 4E). This trend was accompanied by a significant reduction in nucleotide diversity of the plasma virus population in the high-potency HIG group, assessed at both the gB and gL loci (Figure 5 and Supplemental Figure 7). Thus, we hypothesize that in the setting of antibody-mediated immune pressure, the number of unique viral variants is restricted following primary infection and systemic replication is inhibited, resulting in decreased viral transmission to the fetus.

The placental transcriptome was examined to investigate whether HIG-mediated protection against congenital infection occurs via gene regulation at the level of the placenta/decidua. Intriguingly, RhCMV infection impacted the transcription profile of placental/decidual cells to a much greater degree than HIG treatment or fetal abortion, suggesting that HIG predominantly mediated protection through its impact on maternal systemic virus replication. The preferential activation of innate over adaptive immune processes in RhCMV-infected placenta (Figure 7D) is perhaps due to the tolerogenic environment at the maternofetal interface $(52,53)$. Furthermore, the apparent upregulation of NK cell-specific genes in RhCMV-infected placenta (Figure 7, D and E) may be attributable to either NK cells being the major leukocyte in the decidua during the early stages of pregnancy (54) or to the propensity for NK cells to control CMV infection in vivo $(55,56)$. However, this analysis has several caveats including (a) placental samples were from fullthickness decidua/placenta tissue and therefore lack anatomic specificity and (b) all tissue samples were isolated from $\mathrm{CD}^{+} \mathrm{T}$ cell-depleted animals. As such, we cannot be certain whether the trends observed are broadly applicable outside of this model system. 
Our findings are based on a relatively small cohort of animals $(n=12)$ due to the severely limited availability of breeding, seronegative female monkeys. One consequence of our limited animal population is the need to amplify the biologic effects of placental RhCMV infection through both $\mathrm{CD} 4^{+} \mathrm{T}$ cell depletion and i.v. viral inoculation. Though both these artificial methods distort the reality of natural CMV infection and bias our results in favor of placental transmission, we propose that these interventions strengthen the major finding in this study that preexisting antibody alone (in the absence of cell-mediated immunity) can prevent placental transmission in the setting of acute maternal viremia. One additional limitation to our study design is that, although HIG is known to nonspecifically modulate immune responses to prevent inflammatory disease $(44,57,58)$, we had to limit the number of individual control groups in this study and did not have a cohort of animals infused with non-RhCMV-reactive HIG. Because of this deficiency, we cannot definitively rule out nonspecific immunomodulatory effects of the infused HIG, though all infusion groups had similar cytokine expression profiles following infection (Supplemental Figure 9). Furthermore, since protection against congenital transmission may have been achieved as a result of a dose effect in the high-potency HIG group versus the standard group, antibody-mediated protection is likely due to anti-viral function rather than immunomodulatory properties of the infused HIG.

Despite these limitations, this is to our knowledge the first primate study to demonstrate that preexisting maternal antibody alone has the potential to block and ameliorate placental CMV transmission. Thus, a maternal vaccine that elicits durable, potently neutralizing humoral immunity could be an ideal intervention to eliminate pediatric disabilities associated with congenital HCMV infection. Based on our findings we hypothesize that the prevention of maternal HCMV acquisition (i.e., sterilizing immunity), which has been the target of several phase II vaccine trials $(12,13)$, may not be the most appropriate measurable outcome for a congenital HCMV vaccine that ultimately seeks a reduction in the frequency of congenital HCMV transmission. Since we observed that antibody-mediated protection against congenital HCMV was associated with a decrease in maternal systemic viral load and constrained viral diversity, these surrogate markers could potentially serve as clinical study endpoints. Subsequent studies should investigate the epitope specificity and properties of protective immunoglobulins for future rational design of a maternal congenital HCMV vaccine.

\section{Methods}

Animals. Indian-origin rhesus macaques were housed at the Tulane Primate National Research Center and maintained in accordance with institutional and federal guidelines for the care and use of laboratory animals (59). All females were from the expanded specific pathogen-free (eSPF) colony and confirmed to be RhCMV seronegative by whole-virion ELISA screening for RhCMV-specific IgM and IgG plasma antibodies. RhCMV-seronegative males and females were then cohoused and females were screened every 3 weeks for pregnancy via abdominal ultrasound. All animals in this study were between 4 and 9 years of age at the time of pregnancy/enrollment $(\mathrm{JC65}=4.7$, GI73 $=8.8$, IM67 $=5.7$, JM52 $=$ 4.6, HR73 = 7.6, HD82 = 8.7, HD79 = 8.7, GM04 = 8.8, and HD79 = 8.7 years old). Gestational dating was performed by sonography, and was based on gestational sac size (average of 3 dimensions) and crown-rump length of the fetus. At week 7 of gestation, $12 \mathrm{RhCMV}$-seronegative dams were administered an i.v. $50 \mathrm{mg} / \mathrm{kg}$ dose of recombinant rhesus $\mathrm{CD}^{+} \mathrm{T}$ cell-depleting antibody (CD4R1 clone; NIH Nonhuman Primate Reagent Resource)

One week following $\mathrm{CD} 4^{+} \mathrm{T}$ cell depletion, 3 seronegative dams were administered a single dose (100 $\mathrm{mg} / \mathrm{kg}$ ) of HIG produced from the plasma of RhCMV-seropositive monkeys screened by RhCMV wholevirion IgG-binding ELISA $\left(\mathrm{IC}_{50}=22.64 \mu \mathrm{g} / \mathrm{ml}\right) 1$ hour prior to i.v. challenge with a mixture of $\mathrm{RhCMV}$ strains 180.92 (originally isolated from the lung of an immune-compromised rhesus macaque) (30) and UCD52/59 (originally isolated from urine of seropositive monkeys) (31). Each monkey was administered $2 \times 10^{6} 50 \%$ tissue culture infective dose $\left(\mathrm{TCID}_{50}\right) \mathrm{RhCMV} 180.92$ in one arm and $1 \times 10^{6} \mathrm{TCID}_{50}$ each of RhCMV UCD52 and UCD59 in the other arm, diluted in serum-free RPMI. The remaining 3 dams received a dose-optimized ( 2 dose) regimen of a potently neutralizing HIG product purified from $\mathrm{RhC}$ MV-seropositive monkeys with a plasma neutralization titer in epithelial cells greater than 1,000 $\left(\mathrm{IC}_{50}=\right.$ $5.88 \mu \mathrm{g} / \mathrm{ml})$ at 1 hour prior to i.v. RhCMV inoculation $(150 \mathrm{mg} / \mathrm{kg})$ and 3 days after infection $(100 \mathrm{mg} /$ $\mathrm{kg}$ ), respectively. This dose-optimized regimen was based on pharmacokinetic modeling of the neutralization activity from the standard group (Supplemental Figure 4). Six dams were noninfused control animals (including 4 historical controls) (24). 
Blood draws were performed on the standard potency-infused animals on the following days after infection/infusion: $0,2,4,7,10,14,21,28,35$, and 42 . The high-potency-infused animals were sampled at days $0,1,2,3,4,7,10,14,21,28,35,42,49,56,63,70,77$, and 84. Amniotic fluid (by amniocentesis), urine (by clean pan collection), and saliva (by oral saline wash), were also collected on all animals on a weekly to biweekly intervals until fetal harvest. In the case of fetal abortion, placental and fetal products were obtained from the cage and fixed in formalin for H\&E staining and immunohistochemistry or frozen. Fetal growth was monitored by measuring biparietal diameter and femur length during weekly to biweekly fetal sonography over the course of gestation. Sonography was also used to screen for signs of congenital RhCMV-associated sequelae. The fetuses of dams in the standard HIG group were harvested at 6 weeks following infection, and those of the high-potency HIG group at 12 weeks following infection. The standard and high-potency HIG groups were followed for different periods of time because we did not observe any fetal abortion in the first group (standard HIG), and therefore wanted to ensure in the subsequent year that HIG treatment did not simply delay fetal abortion.

IgG purification for HIG preparations. Seropositive rhesus monkey plasma donors were screened by either high-OD binding against UCD52 virions (top 50\% seropositive OD; standard HIG) or by epithelial cellneutralization titer $\left(\mathrm{ID}_{50}>1,000\right.$; high-potency $\left.\mathrm{HIG}\right)$. Donor plasma was mixed with an equal volume of Protein G IgG Binding Buffer (Pierce) and spun at 5,000 $\mathrm{g}$ for 25 minutes at room temperature. Pellets were discarded and supernatants were filter sterilized through a $0.22-\mu \mathrm{m}$ filter and used for purification. The sera mixture was passed through a gravity column packed with Protein G Plus Agarose beads (Pierce). Beads were washed with 5 column volumes (CVs) of IgG Binding Buffer and eluted with 2 CVs of IgG Elution Buffer. Eluted IgG was immediately neutralized with $1 \mathrm{M}$ Tris buffer, $\mathrm{pH}$ 8. Beads were washed with 5 CVs of IgG Binding Buffer. Serum was passed through Protein G Plus Agarose beads 10 times and IgG was eluted each time. All IgG elutions were concentrated and buffer exchanged into 1× PBS using Millipore Amicon 30K filters (Fisher Scientific). Final preparations were tested by ELISA for the presence of bacterial endotoxin. See quality control sheet for high-potency HIG preparation in Supplemental Figure 3.

Pharmacokinetic modeling for dose-optimized HIG infusion. Plasma concentration-time data of IgG in rhesus monkeys were adjusted using the baseline value of IgG for each individual animal and analyzed using WinNonlin (version 6.3.0; Certara). One- and 2-compartment PK models with first-order elimination were evaluated to characterize the observed data. PK parameters including clearance (CL), volume of distribution $(V)$, and half-life associated with the elimination phase $\left(T_{1 / 2, \beta}\right)$ were estimated for each animal. Simulations for optimizing dosing regimen in the second PK study were performed to ensure IgG concentrations above $1-1.2 \mathrm{mg} / \mathrm{ml}\left(\sim \mathrm{ID}_{50}>1,000\right)$ for 2 weeks after initiating treatment.

Cell culture and virus growth. Telomerized rhesus fibroblasts (TeloRFs) were maintained in Dulbecco's modified Eagle medium (DMEM) containing 10\% FCS, $2 \mathrm{mM}$ L-glutamine, $50 \mathrm{U} / \mathrm{ml}$ penicillin, $50 \mu \mathrm{g} /$ $\mathrm{ml}$ each streptomycin and gentamicin, and $100 \mu \mathrm{g} / \mathrm{ml}$ geneticin G418 (Invitrogen). Immortalized TeloRFs were used for in vitro assays and viral growth since they (a) robustly support viral replication indefinitely (i.e., without issues of cellular senescence) and (b) facilitate standardization/consistency between assays (60). Monkey kidney epithelial (MKE) cells were cultured in DMEM-F12 supplemented with 10\% FCS, $2 \mathrm{mM}$ L-glutamine, $1 \mathrm{mM}$ sodium pyruvate, $50 \mathrm{U} / \mathrm{ml}$ penicillin, $50 \mu \mathrm{g} / \mathrm{ml}$ each streptomycin and gentamicin, and $5 \mathrm{ml}$ of Epithelial Cell Growth Supplement (ScienCell). Cell lines were tested for mycoplasma contamination every 6 months.

Epithelial cell-tropic UCD52 and UCD59 were propagated on MKE cells for 3-4 passages, and then passaged once on TeloRFs in order to increase viral titer. This single round of fibroblast amplification was intended to minimize any alteration of viral tropism or glycoprotein surface expression. Virus infections were performed with similar media but with $5 \% \mathrm{FCS}$ for 4 hours at $37^{\circ} \mathrm{C}$ and $5 \% \mathrm{CO}_{2}$. Virus was harvested when cells showed $90 \%$ cytopathic effects (CPEs) by cell scraping. For harvest, infected cells were pelleted by low-speed centrifugation, and the supernatant was placed on ice. Cell pellets were then resuspended in infection media and subjected to 3 freeze/thaw cycles. Following centrifugation, supernatants were combined, passed through a $0.45-\mu \mathrm{m}$ filter, overlaid onto a $20 \%$ sucrose cushion, and ultracentrifuged at 70,000 $g$ for 2 hours at $4^{\circ} \mathrm{C}$ using an SW28 Beckman Coulter rotor. Virus pellets were resuspended in DMEM containing $10 \%$ FCS and titered in TeloRFs using the TCID $_{50}$ method of Reed and Muench.

Characterization of $T$ cells by flow cytometry. Maternal peripheral $\mathrm{CD}^{+}$and $\mathrm{CD} 8^{+} \mathrm{T}$ cells were phenotyped by mixing $100 \mu 1$ of EDTA-anticoagulated blood with a pool of fluorescently labeled monoclonal antibodies including CCR7, CD95, CD28, CD4, CD20, CD3, CD8, and CD45 (Supplemental Table 1). 
After a 30 -minute incubation at $4^{\circ} \mathrm{C}$, cells were washed with PBS supplemented with $2 \%$ FCS and pelleted at $100 \mathrm{~g}$ for 5 minutes. Red blood cells were then lysed by adding $1 \times \mathrm{BD}$ lysis buffer for 40 minutes at room temperature. Intact cells were centrifuged at $150 \mathrm{~g}$ for 5 minutes, washed once with PBS containing $2 \%$ FCS, and resuspended in $100 \mu \mathrm{l}$ of PBS with $2 \%$ FCS. Cells were fixed by adding $15 \mu 1$ of $2 \%$ formaldehyde and processed by flow cytometry. Gating of $\mathrm{CD}^{+}$and $\mathrm{CD} 8^{+}$populations/subpopulations was completed in FlowJo as is outlined in Supplemental Figure 1.

Antibody kinetics and glycoprotein binding specificity. Virus-specific IgM and IgG antibody kinetics were measured in maternal plasma by whole-virion ELISAs, and glycoprotein specificity of HIG and maternal plasma samples was measured by ELISA against soluble RhCMV gB protein and RhPC. Plates were coated with antigen diluted in PBS with $\mathrm{Mg}^{2+}$ and $\mathrm{Ca}^{2+}(\mathrm{PBS}+)$ overnight at $4{ }^{\circ} \mathrm{C}$ - either $5,120 \mathrm{PFU} / \mathrm{ml}$ of filtered RhCMV UCD52 virus for whole-virion ELISA or $0.13 \mu \mathrm{g} / \mathrm{ml}$ soluble glycoprotein. Following incubation, plates were blocked for 2 hours with blocking solution (PBS+, 4\% whey protein, $15 \%$ goat serum, $0.5 \%$ Tween-20), and then 3 -fold serial dilutions of plasma (1:30 to $1: 5,314,410)$ were added to the wells in duplicate for 1.5 hours. Plates were then washed twice and incubated for 1 hour with anti-monkey IgM or anti-monkey IgG HRP-conjugated antibodies (Rockland) at a 1:10,000 dilution. After 4 washes, SureBlue Reserve TMB Microwell Peroxidase Substrate (KPL) was added to the wells for 10 minutes, and the reaction was stopped by addition of $1 \% \mathrm{HCl}$ solution. Plates were read at $450 \mathrm{~nm}$. The lower threshold for antibody reactivity was considered to be 3 SDs above the average OD measured for the preinfection, RhCMV-seronegative samples at the starting plasma dilution (1:30). The end-point dilution reported is the highest dilution of plasma that exceeds this positivity threshold.

Neutralization assays. TeloRFs and MKE cells were seeded into 96-well plates and incubated for 2 days at $37^{\circ} \mathrm{C}$ and $5 \% \mathrm{CO}_{2}$ to achieve $100 \%$ confluence. After 2 days, serial dilutions $(1: 10$ to 1:30,000) of heatinactivated rhesus plasma were incubated with RhCMV 180.92 or RhCMV UCD52 (MOI = 1) in $50 \mu 1$ for 45 minutes at $37^{\circ} \mathrm{C}$. The virus/plasma dilutions were then added in duplicate to wells containing TeloRFs or MKE cells, respectively, and incubated at $37^{\circ} \mathrm{C}$ for 2 hours. After washing, cells were incubated at $37^{\circ} \mathrm{C}$ for an additional 16 hours. Infected cells were then fixed for 20 minutes at $-20^{\circ} \mathrm{C}$ with $1: 1$ methanol/acetone, rehydrated in PBS+ 3 times 5 minutes, and processed for immunofluorescence with $1 \mu \mathrm{g} / \mathrm{ml}$ mouse anti-RhCMV IE-1 monoclonal antibody (provided by Daniel Cawley, Oregon Health and Science University, Portland, Oregon, USA) followed by a 1:500 dilution of goat anti-mouse IgG-Alexa Fluor 488 antibody (Millipore). Nuclei were stained with DAPI for 5 minutes (Pierce). Infection was quantified in each well by automated cell counting software using either (a) a Nikon Eclipse TE2000-E fluorescence microscope equipped with a CoolSNAP HQ-2 camera at $\times 10$ magnification or (b) a Cellomics Arrayscan VTI HCS instrument at $\times 10$ magnification. Subsequently, the $\mathrm{ID}_{50}$ was calculated as the sample dilution that caused a $50 \%$ reduction in the number of infected cells compared with wells treated with virus only using the method of Reed and Muench.

Soluble protein bead coupling and antibody depletion. Cyanogen bromide-activated (CNBr-activated) Sepharose beads (GE Healthcare) were rehydrated in $1 \mathrm{mM} \mathrm{HCl}$, and then suspended in coupling buffer $\left(0.1 \mathrm{M} \mathrm{NaHCO}_{3}+0.5 \mathrm{M} \mathrm{NaCl}, \mathrm{pH} 8.3\right)$ at room temperature. Every $100 \mu 1$ of bead slurry was combined with $150 \mathrm{~g}$ soluble RhgB or RhPC ligands. Coupling was allowed to proceed for 1 hour at room temperature on an inversion rotator. Excess soluble protein was washed off with 5 column volumes of coupling buffer. $\mathrm{CNBr}$ unbound active groups were blocked by incubation in $0.1 \mathrm{M}$ Tris $\mathrm{HCl}, \mathrm{pH} 8.0$ for 2 hours at room temperature on an inversion rotator. Protein-bound beads were washed with 3 cycles of alternating $\mathrm{pH}$ : first $0.1 \mathrm{M}$ acetic acid, $\mathrm{pH} 4.0$, followed by $0.1 \mathrm{M}$ Tris- $\mathrm{HCl}, \mathrm{pH}$ 8.0. Beads were suspended in PBS and stored at $4^{\circ} \mathrm{C}$. For depletion of antibodies for a given specificity, $100 \mu 1$ of protein-coupled bead slurry was loaded into a spin microelution column (Pierce, TFS). Plasma (50 $\mu$ l) was filtered $(0.22$ $\mu \mathrm{m})$ and then loaded into the column. Plasma was centrifuged through the column 5 times without elution between steps. Bound IgG was eluted using 0.2 M glycine elution buffer, $\mathrm{pH}$ 2.5. The column was recalibrated by washing with 3 cycles of solutions of alternating $\mathrm{pH}$ (as described above). Adequate and specific depletion of plasma was confirmed by ELISA against the depleted protein $\left(>75 \%\right.$ change in $\mathrm{EC}_{50}$ against depleted epitope; $<20 \%$ change in $\mathrm{EC}_{50}$ against nondepleted epitope).

Tissue processing and staining. Standard immunoperoxidase staining for RhCMV was performed on formalin-fixed, paraffin-embedded sections of multiple tissues. Tissue sections were deparaffinized in xylene, rehydrated in graded alcohol, and subsequently blocked with $0.3 \%$ hydrogen peroxide in PBS for 
30 minutes. Pretreatment involved microwaving for 20 minutes in $0.01 \%$ citrate buffer (Vector Laboratories), followed by 45 minutes of cooling at room temperature. Following pretreatment, an avidin-biotin block (Invitrogen) and protein block with 10\% normal goat serum (NGS) were conducted on all sections. A wash of Tris-buffered saline with $0.5 \%$ Tween-20 followed each step. Sections were incubated with anti-RhCMV IE1 polyclonal rabbit sera (provided by P.A.B., UC Davis) at a 1:1,600 dilution for 30 minutes at room temperature. Slides were then incubated with biotinylated goat anti-rabbit IgG (Invitrogen) at a 1:200 dilution in NGS for 30 minutes at room temperature. This was followed by a 30 -minute incubation at room temperature with R.T.U. Vectastain ABC (Vector Laboratories). Immunolabeling was visualized using diaminobenzidine and counterstained with Mayer's hematoxylin. Irrelevant, isotype-matched primary antibodies were used in place of the test antibody as negative controls in all immunohistochemical studies. Positive control tissues consisted of archived rhesus macaque lung and testis from an RhCMVseropositive animal.

Viral DNA isolation and assessment of viral load. RhCMV viral load was measured in plasma, amniotic fluid, saliva, urine, and tissues by quantitative RhCMV-specific PCR following DNA extraction. Urine and mouth washes were concentrated using Ultracel YM-100 filters (Amicon) and frozen for subsequent DNA extraction. DNA was extracted from urine using the QIAmp RNA minikit (Qiagen), from amniotic fluid/saliva/plasma by using the QIAmp DNA minikit, and from snap-frozen tissue (10-25 mg) after overnight Proteinase K digestion using the DNeasy Blood and Tissue kit (Qiagen). RhCMV DNA was quantified by real-time PCR using the 5'-GTTTAGGGAACCGCCATTCTG-3' forward primer, 5'-GTATCCGCGTTCCAATGCA-3' reverse primer, and 5'-FAM-TCCAGCCTCCATAGCCGGGAAGG-TAMRA-3' probe, which amplify and detect a 108-bp region of the RhCMV IE1 gene (61). Between 5 and $10 \mathrm{ng}$ of DNA was added as template to $50 \mu 1$ of $1 \times$ PCR mixture containing 300 $\mathrm{nM}$ of each primer, $100 \mathrm{nM}$ probe, $2 \mathrm{mM} \mathrm{MgCl}_{2}, 200 \mu \mathrm{M}$ each of dATP, dCTP, and dGTP, $400 \mu \mathrm{M}$ dUTP, $0.01 \mathrm{U} / \mu 1$ Amperase $\mathrm{UNG}, 0.025 \mathrm{U} / \mu 1$ Taq polymerase, and reaction buffer that includes passive reference dye ROX. PCR conditions consisted of an initial 2-minute cycle at $50^{\circ} \mathrm{C}$ followed by 10 minutes at $95^{\circ} \mathrm{C}$, and then 45 cycles of denaturation at $95^{\circ} \mathrm{C}$ for 15 seconds and combined annealing/ extension at $60^{\circ} \mathrm{C}$ for 1 minute. Data are expressed as copies per milliliter for plasma/amniotic fluid and as copies per microgram input DNA for tissues/saliva/urine. RhCMV DNA was quantified using a standard consisting of a plasmid containing the entire RhCMV IE1 gene. RhCMV DNA was detected with a linear dynamic range from $10^{0}$ to $10^{6}$ copies in the presence of genomic DNA.

$S N A P P$. Viral DNA was isolated from plasma using the High Pure Viral Nucleic Acid kit (Roche Life Science). Hypervariable regions $400 \mathrm{bp}$ in length within $\mathrm{gB}$ and gL were amplified in duplicate by a single round of PCR using 5'-AAGTGCTCGAAGGGCTTCTC-3' and 5'-TCTGGACATTGATCCGCTGG-3' for the $\mathrm{gB}$ region and 5'-GCGCGGCACACATTATCTAC-3' and 5'-GGTGAGTGCTGCTGTTTTGG-3' for the $\mathrm{gL}$ region. Overhang regions were conjugated to primer locus-specific sequences for subsequent Illumina index primer addition and sequencing: forward primer overhang = 5'-TCGTCGGCAGCGTCAGATGTGTATAAGAGACAG-[locus]-3' and reverse primer overhang = 5'-GTCTCGTGGGCTCGGAGATGTGTATAAGAGACAG-[locus]-3'. Approximately $10 \mathrm{ng}$ DNA was added as template to $50 \mu 1$ of $1 \times$ PCR mixture containing $100 \mathrm{nM}$ of each primer, $2 \mathrm{mM} \mathrm{MgCl}_{2}, 200 \mu \mathrm{M}$ each of dNTP mix (Qiagen), and $0.025 \mathrm{U} / \mu 1$ Phusion Taq polymerase. PCR conditions consisted of an initial 2-minute denaturation at $98^{\circ} \mathrm{C}$, followed by the minimum number of PCR cycles to achieve adequate amplification $\left(98^{\circ} \mathrm{C}\right.$ for 10 seconds, $65^{\circ} \mathrm{C}$ for 30 seconds, and $72^{\circ} \mathrm{C}$ for 30 seconds), and then a final $72^{\circ} \mathrm{C}$ extension for 10 minutes. Products were purified using Agencourt AMPure XP beads (Beckman Coulter), and then Illumina Nextera XT index primers were added by 15 cycles of amplification. The resultant PCR product was gel purified using ZR-96 Zymoclean Gel DNA Recovery Kit (Zymogen). The molar amount of each sample was normalized by real-time PCR using the KAPA library amplification kit (KAPA Biosystems). The library of individual amplicons was pooled together, diluted to an end concentration of $10 \mathrm{pM}$, combined with $20 \%$ V3 PhiX (Illumina), and then sequenced on Illumina Miseq using a 600-cycle V3 cartridge (Illumina). For each primer set we confirmed that there was no significant primer bias through mixing viral DNA in known ratios and applying the SNAPP technique (Supplemental Figure 6).

SNAPP amplicon reconstruction and nucleotide diversity. Sequences of the targeted regions on the $\mathrm{gB}$ and $\mathrm{gL}$ genes were reconstructed by merging the paired-reads sets using the PEAR software under default parameters (62). The fused reads were then filtered using the extractor tool from the SeekDeep pipeline (http:// baileylab.umassmed.edu/seekdeep), filtering sequences according to their length, overall quality scores, and presence of the primer sequences. To homogenize the number of sequences per sample due to variable 
coverage, a random set of at most 10,000 sequences was retrieved from each sample to estimate strain frequency and nucleotide diversity. The number of sequences analyzed per sample varied from 2,000-10,000, depending on the sample coverage. Next, haplotype reconstruction was performed using the qluster tool from SeekDeep, which accounts for possible sequencing errors by collapsing fragments with mismatches at low-quality positions. For each given sample, the haplotypes had to be present in 2 sample replicates to be taken into account. Each haplotype was assigned to 1 of the 3 inoculated viral strains by first calculating the nucleotide distance (nucleotide substitutions) between the haplotype and the reference viral strain, and then assigning the haplotype to the strain with the shortest nucleotide distance. Nucleotide diversity was computed as the average distance between each possible pair of sequences belonging to the same reference strain (63). Historical control animal 274-98 (24) was omitted from this analysis (Figure 5 and Supplemental Figure 7) because it was only inoculated with a single RhCMV strain (180.92).

Placental RNA isolation and microarray. Tissue sections weighing approximately $30 \mathrm{mg}$ were obtained from flash frozen, full-thickness rhesus placenta/decidua, lysed using a TissueLyser LT (Qiagen), and then total RNA extracted using an RNeasy Mini Kit (Qiagen). RNA was assessed for quality with Agilent 2100 Bioanalyzer G2939A (Agilent Technologies) and Nanodrop 8000 spectrophotometer (Thermo Scientific/Nanodrop) and confirmed to have $\mathrm{A}_{260} / \mathrm{A}_{280}$ greater than $1.8, \mathrm{~A}_{260} / \mathrm{A}_{230}$ greater than 1.0 , and RIN greater than 7.0. Note that the analysis was not completed for animal HR73 (high-potency HIG infused) because high-quality RNA could not be obtained from the tissue sample. Hybridization targets were prepared with MessageAmp Premier RNA Amplification Kit (Applied Biosystems/Ambion) from total RNA, hybridized to Affymetrix GeneChip Rhesus Macaque Genome arrays in an Affymetrix GeneChip hybridization oven 645, washed in an Affymetrix GeneChip Fluidics Station 450, and scanned with an Affymetrix GeneChip Scanner 7G according to standard Affymetrix GeneChip Hybridization, Wash, and Stain protocols. Affymetrix microarray data were initially processed, underwent QC, and were normalized with the robust multi-array average method using the affy (64) Bioconductor (65) package from the $R$ statistical programming environment ( $R$ studio version 0.99 .902 ). Differential expression was carried out using a moderated t-statistic from the limma (66) package. In an attempt to minimize the effect of gestational age on our transcriptome analysis, we corrected for this variable by using a linear model that included time as a covariate (gene expression $\approx$ condition + time). The FDR was used to control for multiple hypothesis testing. Gene set enrichment analysis (67) was performed to identify differentially regulated pathways and gene ontology terms for each of the comparisons performed. Gene sets specific for innate/adaptive immunity, myeloid/lymphoid lineages, and immune cells were obtained from previous reports (35) and supplemented from the literature such that each comparison group had more than 25 characteristic genes (gene lists in Supplemental Dataset 1). Gene networks were constructed using NatureAnalyst (68), with first-order gene interactions inferred from the InnateDB interactome (Figure 7B) (69). Genes related to NK cell function (Supplemental Table 5) were determined using Qiagen's Integrated Pathway Analysis (IPA) (NK cell activation and movement gene lists) and supplemented with those from the KEGG database (NK cell mediated cytotoxicity gene list) (70).

Statistics. Comparisons of peak viremia and shedding (Figure 4, E, G, and H) in monkeys from each group were performed using the exact Wilcoxon test. The correlation between peak plasma viral load and initial amniotic fluid viral load (Figure 4F) was calculated with the Spearman correlation. The BenjaminiHochberg FDR $P$ value correction was used to correct for multiple comparisons (71). For sequence diversity analysis (Figure 5 and Supplemental Figure 7), UCD52 diversity was compared between treatment groups because it was the dominant strain replicating in plasma. First, median UCD52 nucleotide diversity was calculated for each animal. Median values were compared first by Kruskal-Wallis test $(P=0.036)$, followed by post-hoc Mann-Whitney $U$ test. Analysis of survival curves between standard HIG group and high-potency HIG-infusion group was completed with the exact log-rank test based on the Heinze macro (Figure 6, A and B) (33). Statistical analysis was performed with SAS v9.4 (SAS Institute). A $P$ value less than 0.05 (2-tailed) was considered significant for all analyses.

Study approval. The animal protocol was approved by the Tulane University and the Duke University Medical Center IACUCs.

Data and materials availability. SNAPP sequence data used in this manuscript are available in the NCBI Sequence Read Archive (SRA) (PRJNA386504, https://www.ncbi.nlm.nih.gov/bioproject/PRJNA386504). Placental transcriptome microarray data are available in the NCBI's Gene Expression Omnibus (GEO GSE87395; http://www.ncbi.nlm.nih.gov/geo/query/acc.cgi?acc=GSE87395). 


\section{Author contributions}

AK and SRP designed the research. CSN, DT, KMB, MG, RB, XA, and LS performed the research. CSN, DVC, RB, XA, HI, HW, and MC analyzed data. AD, FC, FW, DJD, NV, MW, PAB, MCW, and KK contributed analytic tools/expertise/reagents. CSN, AK, and SRP wrote the manuscript.

\section{Acknowledgments}

The authors would like to recognize David Corcoran and the Duke Sequencing and Genomic Technologies Shared Resource for technical support, microarray data management, and feedback on the generation of the microarray data reported in this manuscript. Additionally, we would like to thank Xinzhen Yang and Pfizer Inc. for the gift of research materials. Finally, the excellent veterinary care and conduct of animal studies by the faculty and staff of the Departments of Veterinary Medicine and Collaborative Research at the Tulane National Primate Research Center (TNPRC) are gratefully acknowledged. The CD4 ${ }^{+} \mathrm{T}$ celldepleting antibody used in these studies was provided by the NIH Nonhuman Primate Reagent Resource (R24 OD010976, and NIAID contract HHSN 272201300031C). This work was supported by an NIH/ NICHD Director's New Innovator grant to S.R.P. (DP2HD075699), the TNPRC base grant NIH/NCRR (OD011104), NIH/NIAID grants to D.J.D. and P.A.B. (R01AI103960, R01AI63356), and a fellowship grant to C.S.N. (F30HD089577). The funders had no role in study design, data collection and interpretation, decision to publish, or the preparation of this manuscript. The content is solely the responsibility of the authors and does not necessarily represent the official views of the NIH.

Address correspondence to: Sallie R. Permar, 2 Genome Ct., MSRB2 Rm 3061, Durham, North Carolina 27710, USA. Phone: 919.684.2515; Email: sallie.permar@dm.duke.edu. Or to: Amitinder Kaur, 18703 Three Rivers Road, Covington, Louisiana 70433, USA. Phone: 985.871.6530; Email: akaur@tulane.edu.

1. Manicklal S, Emery VC, Lazzarotto T, Boppana SB, Gupta RK. The "silent" global burden of congenital cytomegalovirus. Clin Microbiol Rev. 2013;26(1):86-102.

2. Centers for Disease Control Prevention (CDC). Knowledge and practices of obstetricians and gynecologists regarding cytomegalovirus infection during pregnancy--United States, 2007. MMWR Morb Mortal Wkly Rep. 2008;57(3):65-68.

3. Rasmussen SA, Jamieson DJ, Honein MA, Petersen LR. Zika virus and birth defects--reviewing the evidence for causality. N Engl J Med. 2016;374(20):1981-1987.

4. Centers for Disease Control Prevention (CDC). Impact of expanded newborn screening--United States, 2006. MMWR Morb Mortal Wkly Rep. 2008;57(37):1012-1015.

5. Mussi-Pinhata MM, et al. Birth prevalence and natural history of congenital cytomegalovirus infection in a highly seroimmune population. Clin Infect Dis. 2009;49(4):522-528.

6. Yamamoto AY, et al. Congenital cytomegalovirus infection as a cause of sensorineural hearing loss in a highly immune population. Pediatr Infect Dis J. 2011;30(12):1043-1046.

7. Institute of Medicine (US) Committee to Study Priorities for Vaccine Development. In: Stratton KR, Durch JS, Lawrence RS eds. Vaccines for the 21st Century: A Tool for Decisionmaking. Washington (DC): National Academies Press (US); 2000.

8. Elek SD, Stern H. Development of a vaccine against mental retardation caused by cytomegalovirus infection in utero. Lancet. 1974;1(7845):1-5.

9. Plotkin SA, Farquhar J, Horberger E. Clinical trials of immunization with the Towne 125 strain of human cytomegalovirus. J Infect Dis. 1976;134(5):470-475.

10. Starr SE, Glazer JP, Friedman HM, Farquhar JD, Plotkin SA. Specific cellular and humoral immunity after immunization with live Towne strain cytomegalovirus vaccine. J Infect Dis. 1981;143(4):585-589.

11. Pass RF, et al. A subunit cytomegalovirus vaccine based on recombinant envelope glycoprotein B and a new adjuvant. J Infect Dis. 1999;180(4):970-975.

12. Pass RF, et al. Vaccine prevention of maternal cytomegalovirus infection. N Engl J Med. 2009;360(12):1191-1199.

13. Bernstein DI, et al. Safety and efficacy of a cytomegalovirus glycoprotein B (gB) vaccine in adolescent girls: A randomized clinical trial. Vaccine. 2016;34(3):313-319.

14. Adler SP, et al. A canarypox vector expressing cytomegalovirus (CMV) glycoprotein B primes for antibody responses to a live attenuated CMV vaccine (Towne). J Infect Dis. 1999;180(3):843-846

15. Berencsi $\mathrm{K}$, et al. A canarypox vector-expressing cytomegalovirus (CMV) phosphoprotein 65 induces long-lasting cytotoxic $\mathrm{T}$ cell responses in human CMV-seronegative subjects. J Infect Dis. 2001;183(8):1171-1179.

16. Wussow F, et al. Human cytomegalovirus vaccine based on the envelope gH/gL pentamer complex. PLoS Pathog. 2014;10(11):e1004524.

17. Wloch MK, et al. Safety and immunogenicity of a bivalent cytomegalovirus DNA vaccine in healthy adult subjects. J Infect Dis. 2008;197(12):1634-1642.

18. Jacobson MA, et al. A CMV DNA vaccine primes for memory immune responses to live-attenuated CMV (Towne strain). Vaccine. 2009;27(10):1540-1548.

19. Gardner TJ, Tortorella D. Virion glycoprotein-mediated immune evasion by human cytomegalovirus: a sticky virus makes a 
slick getaway. Microbiol Mol Biol Rev. 2016;80(3):663-677.

20. Lilleri D, Kabanova A, Lanzavecchia A, Gerna G. Antibodies against neutralization epitopes of human cytomegalovirus gH/gL/ pUL128-130-131 complex and virus spreading may correlate with virus control in vivo. J Clin Immunol. 2012;32(6):1324-1331.

21. Lilleri D, et al. Fetal human cytomegalovirus transmission correlates with delayed maternal antibodies to gH/gL/pUL128-130131 complex during primary infection. PLoS One. 2013;8(3):e59863.

22. Boppana SB, Britt WJ. Antiviral antibody responses and intrauterine transmission after primary maternal cytomegalovirus infection. J Infect Dis. 1995;171(5):1115-1121.

23. Revello MG, et al. A randomized trial of hyperimmune globulin to prevent congenital cytomegalovirus. $N$ Engl J Med. 2014;370(14):1316-1326.

24. Bialas KM, et al. Maternal CD4+ $\mathrm{T}$ cells protect against severe congenital cytomegalovirus disease in a novel nonhuman primate model of placental cytomegalovirus transmission. Proc Natl Acad Sci U S A. 2015;112(44):13645-13650.

25. de Rijk EPCT, Van Esch E. The macaque placenta - a mini-review. Toxicol Pathol. 2008;36(Suppl7):108S-118S.

26. Hansen SG, Strelow LI, Franchi DC, Anders DG, Wong SW. Complete sequence and genomic analysis of rhesus cytomegalovirus. J Virol. 2003;77(12):6620-6636.

27. Yue Y, Barry PA. Rhesus cytomegalovirus a nonhuman primate model for the study of human cytomegalovirus. Adv Virus Res 2008;72:207-226.

28. Lockridge KM, et al. Primate cytomegaloviruses encode and express an IL-10-like protein. Virology. 2000;268(2):272-280.

29. Powers CJ, Früh K. Signal peptide-dependent inhibition of MHC class I heavy chain translation by rhesus cytomegalovirus. PLoS Pathog. 2008;4(10):e1000150.

30. Kaur A, Daniel MD, Hempel D, Lee-Parritz D, Hirsch MS, Johnson RP. Cytotoxic T-lymphocyte responses to cytomegalovirus in normal and simian immunodeficiency virus-infected rhesus macaques. $J$ Virol. 1996;70(11):7725-7733.

31. Oxford $\mathrm{KL}$, et al. Open reading frames carried on $\mathrm{UL} / \mathrm{b}^{\prime}$ are implicated in shedding and horizontal transmission of rhesus cytomegalovirus in rhesus monkeys. J Virol. 2011;85(10):5105-5114.

32. Revello MG, Sarasini A, Zavattoni M, Baldanti F, Gerna G. Improved prenatal diagnosis of congenital human cytomegalovirus infection by a modified nested polymerase chain reaction. J Med Virol. 1998;56(1):99-103.

33. Heinze G, Gnant M, Schemper M. Exact log-rank tests for unequal follow-up. Biometrics. 2003;59(4):1151-1157.

34. Tabata T, Petitt M, Fang-Hoover J, Zydek M, Pereira L. Persistent cytomegalovirus infection in amniotic membranes of the human placenta. Am J Pathol. 2016;186(11):2970-2986.

35. Smith CL, et al. Identification of a human neonatal immune-metabolic network associated with bacterial infection. Nat Commun. 2014;5:4649.

36. Fowler KB, Stagno S, Pass RF. Maternal immunity and prevention of congenital cytomegalovirus infection. JAMA. 2003;289(8):1008-1011.

37. Bratcher DF, et al. Effect of passive antibody on congenital cytomegalovirus infection in guinea pigs. J Infect Dis. 1995;172(4):944-950.

38. Bourne N, Schleiss MR, Bravo FJ, Bernstein DI. Preconception immunization with a cytomegalovirus (CMV) glycoprotein vaccine improves pregnancy outcome in a guinea pig model of congenital CMV infection. J Infect Dis. 2001;183(1):59-64.

39. Chatterjee A, Harrison CJ, Britt WJ, Bewtra C. Modification of maternal and congenital cytomegalovirus infection by antiglycoprotein b antibody transfer in guinea pigs. J Infect Dis. 2001;183(11):1547-1553.

40. Auerbach MR, et al. A neutralizing anti-gH/gL monoclonal antibody is protective in the guinea pig model of congenital CMV infection. PLoS Pathog. 2014;10(4):e1004060.

41. Nigro G, Adler SP, La Torre R, Best AM, Congenital Cytomegalovirus Collaborating Group. Passive immunization during pregnancy for congenital cytomegalovirus infection. NEngl J Med. 2005;353(13):1350-1362.

42. Visentin S, et al. Early primary cytomegalovirus infection in pregnancy: maternal hyperimmunoglobulin therapy improves outcomes among infants at 1 year of age. Clin Infect Dis. 2012;55(4):497-503.

43. Nigro G, et al. Immunoglobulin therapy of fetal cytomegalovirus infection occurring in the first half of pregnancy--a casecontrol study of the outcome in children. J Infect Dis. 2012;205(2):215-227.

44. Maidji E, et al. Antibody treatment promotes compensation for human cytomegalovirus-induced pathogenesis and a hypoxialike condition in placentas with congenital infection. Am J Pathol. 2010;177(3):1298-1310.

45. Cekinović D, et al. Passive immunization reduces murine cytomegalovirus-induced brain pathology in newborn mice. $J$ Virol. 2008;82(24):12172-12180.

46. Planitzer CB, Saemann MD, Gajek H, Farcet MR, Kreil TR. Cytomegalovirus neutralization by hyperimmune and standard intravenous immunoglobulin preparations. Transplantation. 2011;92(3):267-270.

47. Fouts AE, Chan P, Stephan JP, Vandlen R, Feierbach B. Antibodies against the gH/gL/UL128/UL130/UL131 complex comprise the majority of the anti-cytomegalovirus (anti-CMV) neutralizing antibody response in CMV hyperimmune globulin. $J$ Virol. 2012;86(13):7444-7447.

48. Lilleri D, et al. Fetal human cytomegalovirus transmission correlates with delayed maternal antibodies to gH/gL/pUL128-130131 complex during primary infection. PLoS One. 2013;8(3):e59863.

49. Coleman S, Choi KY, Root M, McGregor A. A homolog pentameric complex dictates viral epithelial tropism, pathogenicity and congenital infection rate in guinea pig cytomegalovirus. PLoS Pathog. 2016;12(7):e1005755.

50. Wang D, et al. Quantitative analysis of neutralizing antibody response to human cytomegalovirus in natural infection. Vaccine. 2011;29(48):9075-9080.

51. Revello MG, Zavattoni M, Sarasini A, Percivalle E, Simoncini L, Gerna G. Human cytomegalovirus in blood of immunocompetent persons during primary infection: prognostic implications for pregnancy. J Infect Dis. 1998;177(5):1170-1175.

52. Koga K, Mor G. Toll-like receptors at the maternal-fetal interface in normal pregnancy and pregnancy disorders. Am J Reprod Immunol. 2010;63(6):587-600.

53. Racicot K, Kwon JY, Aldo P, Silasi M, Mor G. Understanding the complexity of the immune system during pregnancy. Am $J$ Reprod Immunol. 2014;72(2):107-116.

54. Male V, Sharkey A, Masters L, Kennedy PR, Farrell LE, Moffett A. The effect of pregnancy on the uterine NK cell KIR reper- 
toire. Eur J Immunol. 2011;41(10):3017-3027.

55. Min-Oo G, Lanier LL. Cytomegalovirus generates long-lived antigen-specific NK cells with diminished bystander activation to heterologous infection. J Exp Med. 2014;211(13):2669-2680.

56. Kuijpers TW, Baars PA, Dantin C, van den Burg M, van Lier RA, Roosnek E. Human NK cells can control CMV infection in the absence of T cells. Blood. 2008;112(3):914-915.

57. Adler SP, Nigro G. Findings and conclusions from CMV hyperimmune globulin treatment trials. J Clin Virol. 2009;46 Suppl 4:S54-S57.

58. Cheeran MC, Lokensgard JR, Schleiss MR. Neuropathogenesis of congenital cytomegalovirus infection: disease mechanisms and prospects for intervention. Clin Microbiol Rev. 2009;22(1):99-126.

59. National Research Council (US) Committee for the Update of the Guide for the Care and Use of Laboratory Animals. Guide for the Care and Use of Laboratory Animals. Washington DC: National Academies Press; 2011.

60. Chang WL, Kirchoff V, Pari GS, Barry PA. Replication of rhesus cytomegalovirus in life-expanded rhesus fibroblasts expressing human telomerase. J Virol Methods. 2002;104(2):135-146.

61. Kaur A, Hale CL, Noren B, Kassis N, Simon MA, Johnson RP. Decreased frequency of cytomegalovirus (CMV)-specific CD4 ${ }^{+}$ T lymphocytes in simian immunodeficiency virus-infected rhesus macaques: inverse relationship with CMV viremia. $J$ Virol. 2002; 76(8):3646-3658.

62. Zhang J, Kobert K, Flouri T, Stamatakis A. PEAR: a fast and accurate Illumina Paired-End reAd mergeR. Bioinformatics. 2014;30(5):614-620.

63. Nelson CW, Hughes AL. Within-host nucleotide diversity of virus populations: insights from next-generation sequencing. Infect Genet Evol. 2015;30:1-7.

64. Gautier L, Cope L, Bolstad BM, Irizarry RA. affy--analysis of Affymetrix GeneChip data at the probe level. Bioinformatics. 2004;20(3):307-315

65. Gentleman RC, et al. Bioconductor: open software development for computational biology and bioinformatics. Genome Biol. 2004;5(10):R80

66. Ritchie ME, et al. limma powers differential expression analyses for RNA-sequencing and microarray studies. Nucleic Acids Res. 2015;43(7):e47

67. Mootha VK, et al. PGC-1alpha-responsive genes involved in oxidative phosphorylation are coordinately downregulated in human diabetes. Nat Genet. 2003;34(3):267-273.

68. Xia J, Gill EE, Hancock RE. NetworkAnalyst for statistical, visual and network-based meta-analysis of gene expression data. Nat Protoc. 2015;10(6):823-844.

69. Breuer K, et al. InnateDB: systems biology of innate immunity and beyond--recent updates and continuing curation. Nucleic Acids Res. 2013;41(Database issue):D1228-D1233.

70. Kanehisa M, Goto S. KEGG: kyoto encyclopedia of genes and genomes. Nucleic Acids Res. 2000;28(1):27-30.

71. Benjamini Y, Hochberg Y. Controlling the false discovery rate: a practical and powerful approach to multiple testing. $J R$ Stat Soc Series B Stat Methodol. 1995;57(1):289-300. 